\title{
Demonstration of Two-dimensional Time-encoded Imaging of Fast Neutrons
}

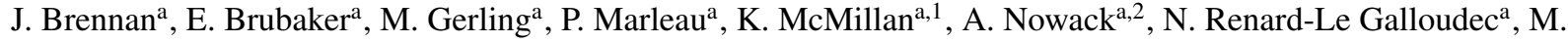 \\ Sweany ${ }^{\mathrm{a}, *}$ \\ ${ }^{a}$ Sandia National Laboratory, Livermore, CA 94550, USA
}

\begin{abstract}
We present a neutron detector system based on time-encoded imaging, and demonstrate its applicability toward the spatial mapping of special nuclear material. We demonstrate that two-dimensional fast-neutron imaging with $2^{\circ}$ resolution at 2 meter stand-off is feasible with only two instrumented detectors.
\end{abstract}

Keywords: fast neutron imaging, time-encoded imaging, special nuclear material detection

\section{Introduction}

Time-encoded imaging (TEI) is an approach to directional fast neutron or gamma detection that has the potential to reduce cost and complexity compared to current imaging systems. TEI is in many ways analogous to coded-aperture imaging, in which the spatial modulation of a particle flux is induced by a fixed mask and detected by a position-sensitive detector. Rather than modulating the particle flux in space, TEI modulates the particle flux in time. The modulated flux is detected by a small number of time-sensitive detectors as opposed to highly pixelated position sensitive detectors in the case of coded-aperture imaging. Essentially, a moving mask that attenuates incoming particles results in a time structure that depends on the source location.

A TEI system addresses serious drawbacks of other neutron and gamma imaging systems. For example, the neutron scatter camera $[1,2]$ and Compton imagers rely on multiple interactions in order to determine the trajectory of incoming particles. Such systems inherently suffer from low efficiency, while requiring multiple planes instrumented with high channel count, position-sensitive detectors. Coded-aperture systems [3] also require high channel count, position sensitive detectors, which can be a source of systematic uncertainty in the detector response. In contrast, a single detector with a time-modulated collimator encodes directional information in the time distribution of detected events.

\footnotetext{
${ }^{*}$ Corresponding author: msweany@ sandia.gov

${ }^{1}$ Currently at the University of California at Los Angeles

${ }^{2}$ Currently at the University of Tennessee at Knoxville
}

We foresee two transformative advantages of TEI. First, a new design space is opened for effective and low-cost imaging systems. Time-encoding based imaging systems have a low channel count, reducing cost and increasing robustness by simplifying system integration, calibration, and reducing systematic uncertainties. Second, the angular resolution of the image reconstruction depends primarily on the collimator design: it is effectively decoupled from the method used for particle detection, which drives the detection efficiency as well as the energy resolution for gamma imaging. Thus, in a time-encoding imager, both angular resolution and energy resolution can be independently optimized. There is, however, a tradeoff between the desired angular resolution of the image and sensitivity to the source. Because a single mask element must completely block a point source from the detector's view, there is a limit to how large the detector(s) can be while maintaining the high imaging resolution yielded by smaller mask elements. Therefore, the system must be optimized for the desired application, taking into account the typical distance from the source.

Finally, there are a number of theoretical and technical challenges associated with a time-modulated mask. In coded-aperture imaging, the mask pattern is typically chosen to minimize correlations: for example, Uniformly Redundant Arrays, or URAs, are based on binary sequences with delta-function autocorrelation functions, or system point-spread functions [4]. Modified Uniformly Redundant Arrays, or MURAs, are binary sequences with the constraint that they have a correlational inverse which is unimodular. Such patterns not only have a delta-function point spread response, but

July 29, 2015 
image with an optimal signal to noise ratio for point-like sources [4].

Neutron sources in the context of nuclear nonprolif- 115 eration applications are generally not ideal: the sources 116 may be extended rather than point-like. In such cases, 117 the optimized signal to noise ratio is not typically 118 achieved with the $\sim 50 \%$ open fraction, or throughput, of 119 MURAs. In addition, the particle source is not infinitely 120 far away, and the effective attenuation of fast neutrons requires thick masks: the image is not the perfect correlation between the source and mask, but includes secondary effects in addition to a background component. For these reasons, a random mask pattern is used for these studies, allowing the freedom to choose an open fraction suited to extended sources. It has been shown that a random pattern is sufficient at limiting artifacts in the reconstructed image, although there is a possibility that correlations exist in long random sequences [5].

Here we report the results of laboratory demonstrations of the TEI concept applied to two-dimensional imaging of distributions of SNM with a prototype system. We first describe the construction of the detector system, calibrations, and data acquisition and analysis in Section 2. Next, measurements and results of three imaging scenarios are presented in Section 3.

\section{Detector System}

The goal of our two-dimensional TEI prototype was to demonstrate proof of feasibility for high resolution image reconstruction. As such, the major driver was to design a mask-detector geometry that achieves a relatively high intrinsic angular resolution. Smaller sensitive detector volumes were chosen for better imaging resolution at the cost of lower sensitivity. Dwell times reported here have the potential to be reduced with a fully optimized system.

The final design has a rotating cylindrical mask consisting of 27 vertical rows of high-density polyethylene (HDPE) mask elements. Each element is an arc segment with an angular width of 2.4 degrees and height of 1.9 $\mathrm{cm}$. The inner diameter of the arc segments is $45 \mathrm{~cm}$, and the outer diameter is $55 \mathrm{~cm}$. The thickness of the elements was chosen to sufficiently moderate neutrons resulting from fission, for which the average scattering ${ }_{134}$ length through HDPE over the relevant energy range is 135 approximately $4.5 \mathrm{~cm}$. With appropriately sized central ${ }_{136}$ detectors, this mask design gives an expected intrinsic ${ }_{137}$ angular resolution of $\sim 2.5^{\circ}$ in both horizontal and ver- ${ }_{138}$ tical dimensions. Each row is constructed from eight 139 $1 / 8$ arc sections machined from a single sheet of HDPE, 140 leaving a 0.125 in thick layer on the inner radius as a ${ }_{141}$ backing to secure each element to the mask. The 216 segments were then adhered together to form a single self-supporting structure. Placed at the center of the rotating mask are two one inch diameter by one inch deep aluminum cells filled with EJ-309 liquid organic scintillator, each coupled to one Hamamatsu H1949-50 two inch diameter photomultiplier tube assembly. A photo of the prototype detector as built is shown in Figure 1.

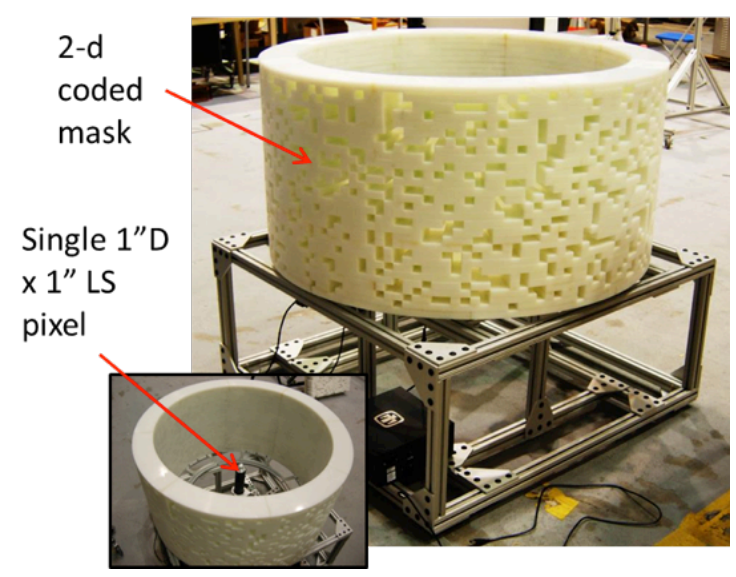

Figure 1: Photograph of the 2-D TEI system. Two central one inch diameter by one inch thick liquid organic scintillator detectors (inset) are surrounded by a rotating high density polyethylene mask. The mask has an inner diameter of $44 \mathrm{~cm}$, an outer diameter of $55 \mathrm{~cm}$, and a height of $50.8 \mathrm{~cm}$.

In a study of mask designs for a traditional coded aperture imaging system, an open fraction of $30 \%$ was determined to be optimal for the extended source distributions that were used in laboratory tests [6]. This open fraction resulted in a total of 2816 mask elements and 1207 apertures. The mask pattern was chosen out of 100 randomly generated pattens: simulated image reconstruction performance was evaluated against several test distributions, and the mask with the most accurate reconstruction and the fewest artifacts was selected. The result is not an optimal pattern, but it was judged to be good enough to demonstrate the feasibility of this technique.

The mask sits on a rotation table driven by an Arcus stepper motor: the drive wheel of the motor is in contact with a rubber gear strip that runs along the inner main wheel of the rotation table. A rotary encoder is attached to the table ring to measure the angle of rotation. Finally, a metal divot attached to the rotation table passes over a switch on the stationary mask frame to mark the end of one rotation. 


\subsection{Electronics and Data Acquisition}

Signals from the PMTs were digitized by a Struck SIS3316, $250 \mathrm{MS} / \mathrm{s}$ desktop digitizer. During the measurements, the detector system continuously buffered data from the SIS3316 digitizers. After a fixed dwell time, the detector was halted and the entire buffer is ${ }^{167}$ written to hard disk. This allowed for close synchro- 168 nization of position information recorded by the en- 169 coder to the data recorded for each time period without 170 having to use multithreaded acquisition software. As a 171 result, the system experienced a dead-time of $\sim 5 \%$ dur- 172 ing read-out.

During measurements, an Arcus controller board continuously read the encoder value, motor pulse value, and the state of the frame switch. When the switch transitioned between off and on values, a digital pin connected to a DG535 pulse generator outputted a NIM compatible signal to the clock timestamp reset of the digitizer. When a new rotation began, the readout event timestamp coincided with a time equal to zero.

\subsection{Calibration}

The Compton pulse height spectrum from a ${ }^{22} \mathrm{Na}$ gamma-ray source was used to match the gains of the two central liquid scintillator cells. The resulting gainmatched spectra are shown in Figure 2.

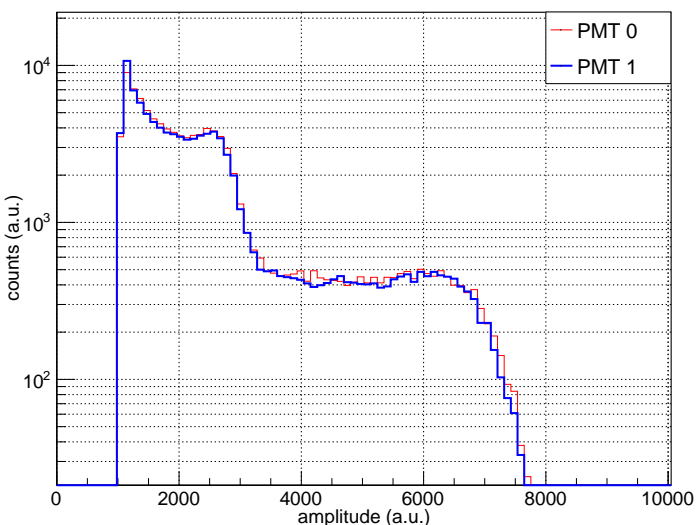

Figure 2: The measured pulse height spectrum from the two liquid 180 scintillator cells using a ${ }^{22} \mathrm{Na}$ gamma source.

Next, calibration measurements were obtained using 183 an AmBe source in order to establish neutron/gamma 184 discrimination cuts based on pulse shape. A pulse shape 185 discrimination (PSD) parameter was defined as the ratio 186 of the tail integral to the total integral, where the be- 187 ginning of the tail window was chosen to maximize the 188 separation between the neutron and gamma bands by optimizing the figure-of-merit FOM:

$$
\mathrm{FOM}=\frac{\mu_{n}-\mu_{\gamma}}{\Gamma_{n}+\Gamma_{\gamma}}
$$

where $\mu_{n, \gamma}$ is the mean of the neutron and gamma bands and $\Gamma_{n, \gamma}$ is the width (FWHM) of the neutron and gamma bands. The total pulse width is 80 samples long (see Figure 3), and the first ten samples are used to establish a baseline. The optimized tail window starts at 30 samples.

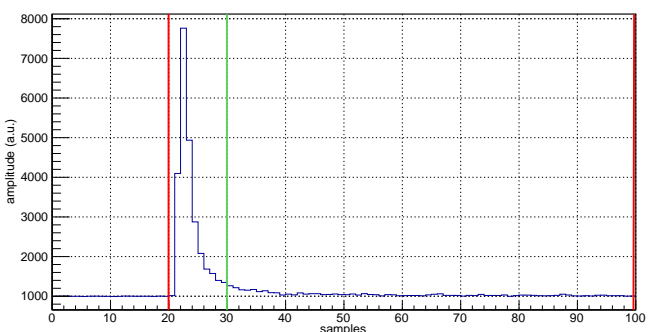

Figure 3: An example waveform with the total pulse window shown in red (20-100 samples) and the start of the tail window shown in green (30 samples). The sample size on the $\mathrm{x}$-axis is $4 \mathrm{~ns}$ wide.

The PSD parameter is plotted against pulse amplitude for each of the PMTs in Figure 4. This PSD parameter distribution was then fit by a double Gaussian in slices of pulse amplitude. The resulting fit parameters were used to construct a likelihood ratio for particle identification, defined as:

$$
\frac{\mathcal{L}_{n}}{\mathcal{L}_{n}+\mathcal{L}_{\gamma}}=\frac{g\left(\mathrm{PSD}, \mu_{n}, \sigma_{n}\right)}{g\left(\mathrm{PSD}, \mu_{n}, \sigma_{n}\right)+g\left(\mathrm{PSD}, \mu_{\gamma}, \sigma_{\gamma}\right)},
$$$$
5 \text { enct }
$$

where $g$ denotes a Gaussian probability density function, and $\mu_{n, \gamma}, \sigma_{n, \gamma}$ are the fitted mean and width, respectively, of the neutron and gamma bands. Finally, a pulse height-dependent neutron selection cut is determined based on the requirement that the likelihood ratio, as defined by Equation (2), is greater than 0.9999 .

\subsection{Analysis}

In offline analysis, the amplitude and PSD parameter were determined from digitized PMT pulses, and neutrons were selected as described above. Each timesorted event was then matched to a rotation angle based on the timestamp of the event and a time-tagged list of encoder positions.

The result of this processing is two arrays (or histograms) of neutron counts vs. rotation angle (see Figure 5), one for each liquid scintillator detector. Due to 

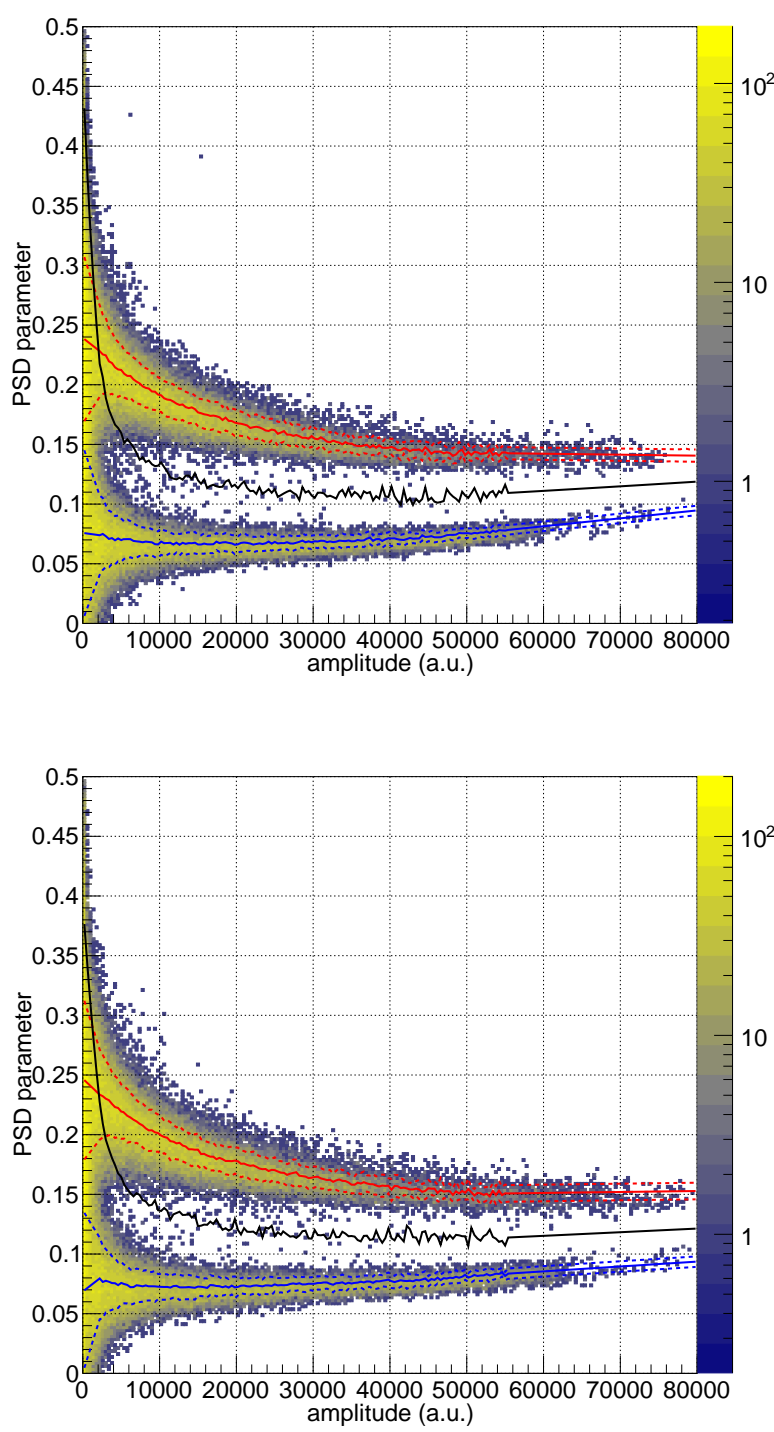

Figure 4: The measured pulse shape parameter vs. pulse amplitude for the two liquid scintillator cells. Neutron events lie within the upper band defined by the red, dotted lines (solid, red line is the ${ }^{216}$ mean and dotted red lines contain $3 \sigma$ deviations from the mean) while 217 gamma/electron events fall in the lower band defined by the blue dot- 218 ted lines (solid, blue line is the mean and the dotted blue lines contain $3 \sigma$ deviations from the mean). The black line between the two distributions represents a $99.99 \%$ Bayesian probability, as determined by Equation (2), of being a neutron.

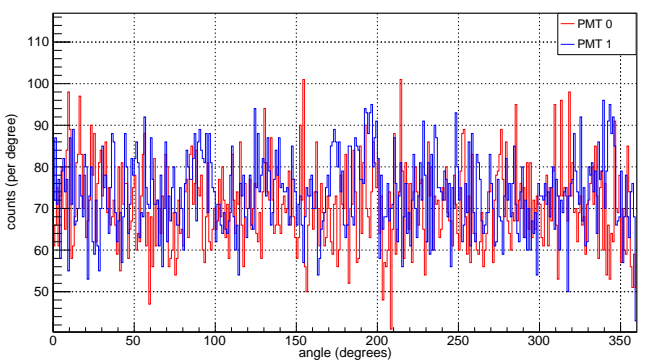

Figure 5: Example data from both photomultiplier tubes after preprocessing. This particular dataset is an hour-long acquisition with the ring source at 2 meters.

uneven amounts of time spent accelerating and decelerating, as well as slippage in the drive wheels, the dwell time as a function of rotation angle is typically not flat. The neutron count at each rotation angle was corrected by dividing by the time spent at each angle read out by the encoder.

Finally, a maximum likelihood expectation maximization (MLEM) [7] algorithm was used to reconstruct a two-dimensional image. In this method, a detector response matrix is used to forward project an assumed neutron source distribution over the two-dimensional space to a predicted set of neutron rate vs. rotation angle measurements. The algorithm then provides an update to the source distribution based on a comparison between the measured and forward-projected values. Each iteration of this updating procedure increases the likelihood that the source distribution produces the measured rates. The detector response matrix is built from the path length of HDPE between a randomly sampled position in both a detector and an arbitrary source pixel. Path lengths are calculated using the TGeoManager class of the ROOT analysis toolkit [8]. The attenuation is then calculated from the average of $10 \mathrm{k}$ path lengths from each source pixel, assuming a neutron attenuation length $4.5 \mathrm{~cm}$, determined by averaging over neutron energies from a ${ }^{252} \mathrm{Cf}$ source.

In Section 3, the results of MLEM iterative image reconstructions are shown. The number of iterations that were used depended on the statistics in each data set. With more neutron counts, more iterations are possible before the statistical fluctuations in the data begin to be amplified, resulting in imaging artifacts. For very highstatistics datasets, statistical and systematic uncertainties in the detector response matrix itself limit the image reconstruction accuracy. The number of iterations was chosen based on the variance of background regions of the image. The ideal number of iterations is taken to 
be just prior to the point where noise in the data or re- 261 sponse matrix begins to drive high-spatial-frequency os- 262 cillations in the reconstructed flat-field background; this 263 point is identified by a steep increase in the variance of 264 reconstructed pixels in the background region. In this 265 work, the point at which this occurs was selected by user 266 judgment. Future work will include the automation of a 267 stopping criterion in the MLEM algorithm.

\section{Measurements and Results}

For all measurements presented in this work one or more matched $35 \mu \mathrm{Ci}{ }^{252} \mathrm{Cf}$ fission sources were placed $2.0 \mathrm{~m}$ from the center of the imaging system. Section 3.1 describe point source measurements, in which the ${ }^{252} \mathrm{Cf}$ sources were held in a stand that allowed their horizontal and vertical positions to be set as desired. For measurements in Sec. 3.2, a ring source was physically approximated by rotating a single ${ }^{252} \mathrm{Cf}$ point source in a circle using an automated rig with its axis oriented toward the center of the imaging system. The source was set to rotate with a period that was a non-integer fraction of the mask rotation period so that given a long enough dwell time, all combinations of source location and mask orientation were sampled. For some of the shorter dwell times presented below this is not guaranteed, although it is deemed to be a sufficiently close analog to a continuous extended source distribution for these tests.

As a preliminary test that the encoder positions were accurately mapped to the expected mask orientation, a ${ }^{252} \mathrm{Cf}$ source was placed $1 \mathrm{~cm}$ from the mask at a height level with one of the PMTs. A comparison of the measured neutron rate modulation as compared to the expected modulation pattern is shown in Figure 6. This provides confidence that the mask alignment and encoder synchronization is accurate.

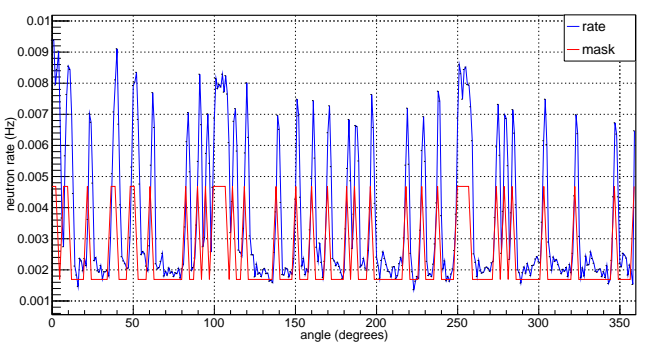

Figure 6: The modulation measured in a single detector with a ${ }^{252} \mathrm{Cf}{ }^{292}$ neutron point source $1 \mathrm{~cm}$ from one row of the mask (blue) overlaid ${ }^{293}$ with the expected modulation pattern (red). Note that the modulation 294 pattern has an arbitrary y-scaling applied.
The TEI system is capable of imaging in an entire 360 degree field of view due to its cylindrical geometry. However, in the following sections, we have limited the reconstruction to the portion of the field of view in which it was known that the source is present. We found that this did not significantly alter the final reconstructed image, but allowed us to reduce the size of the detector response matrix and corresponding time to reconstruct the images. We did not investigate the effect that radiation sources in other regions of the field of view would have on the ability to reconstruct the sources of interest.

\subsection{Point sources}

With mask modulation and registration established, the point source response of the TEI system was explored. Figure 7 shows the MLEM reconstructed image (with 30 MLEM steps) of a single ${ }^{252} \mathrm{Cf}$ point source at $2.0 \mathrm{~m}$ for a $15 \mathrm{~h}$ measurement. The FWHM of this image is smaller than the 1 degree binning used in the image histograms.

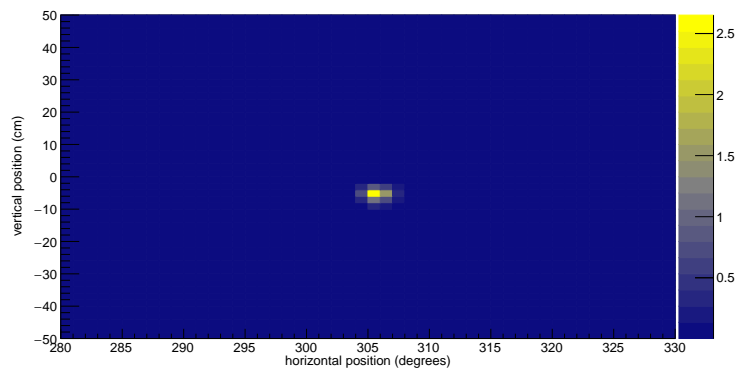

Figure 7: MLEM reconstructed image (30 MLEM steps) of a single ${ }^{252} \mathrm{Cf}$ neutron source at a distance of $2.0 \mathrm{~m}$ with $15 \mathrm{~h}$ dwell time. The $\mathrm{z}$-axis is the rate of neutrons in $\mathrm{Hz}$.

Next, the TEI system's ability to resolve two point sources at varying angular separations was investigated. Figure 8 shows the MLEM reconstructed images of two ${ }^{252} \mathrm{Cf}$ neutron point sources separated by $5^{\circ}$ at a $2.0 \mathrm{~m}$ stand-off. Results are shown for two different vertical positions, each with dwell times of $1 \mathrm{~h}$ and $12 \mathrm{~h}$. These MLEM reconstructed images demonstrate that the resolvable angular separation achievable in $1 \mathrm{~h}$ is less than $5^{\circ}$.

In Figure 9, the limits of small angular separation are explored. Two point sources separated by only $2^{\circ}$ were reconstructed with $2 \mathrm{~h}$ and $24 \mathrm{~h}$ measurement times. In $2 \mathrm{~h}$, the reconstructed image of two point sources is not significantly different than a single point source with twice the strength. However, in $24 \mathrm{~h}$ the statistics are high enough that, though the image does not reveal two 

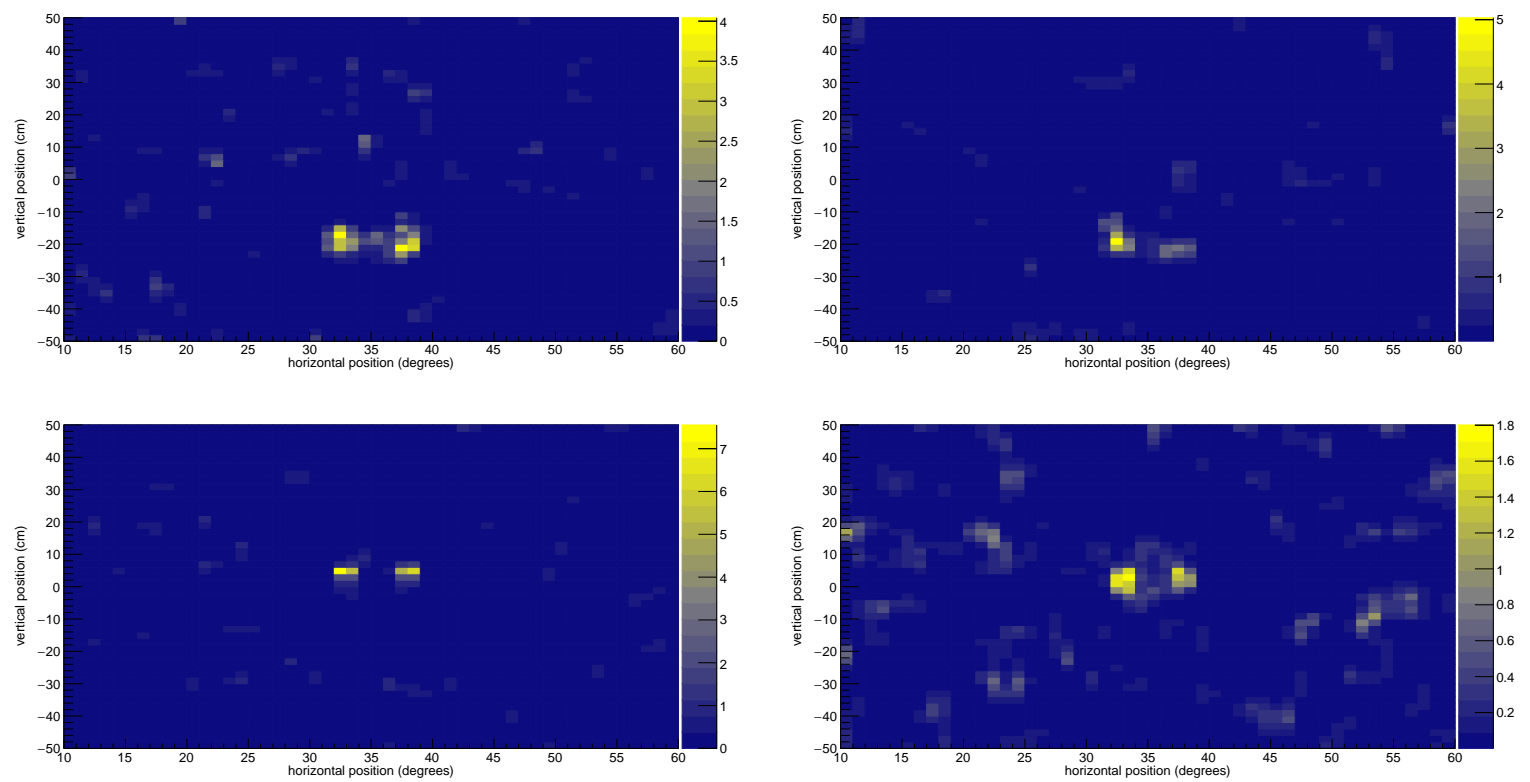

Figure 8: MLEM reconstructions of two ${ }^{252} \mathrm{Cf}$ neutron point sources separated by $5^{\circ}$ at $2.0 \mathrm{~m}$ stand-off. (left column) Two sources at different vertical positions (12 h, 250 MLEM iterations). (right column) Sources at the same positions as in the left column, but only $1 \mathrm{~h}$ dwell (50 MLEM iterations). The $\mathrm{z}$-axis is the rate of neutrons in $\mathrm{Hz}$.

well separated sources, it is significantly different than a single point source (cf. Figure 7).

\subsection{Ring source}

One property of coded aperture imaging is that the signal to noise decreases as multiple and/or extended sources are introduced [5, 9]. Statistically, the signal from one source (or region of an extended source) contributes to the noise for another source (or other region of an extended source). In addition, the source strength is spread out over a larger fraction of the field of view so the intrinsic contrast to background is worse. Therefore, extended source imaging is much more difficult and requires higher statistics to achieve.

To demonstrate the extended source imaging capability of the TEI system, a series of measurements were conducted using the ${ }^{252} \mathrm{Cf}$ ring source described above. In Figure 10, the MLEM image reconstructions for two different vertical displacements of the ring source are shown. In order to provide a sense for how the images develop as a function of dwell time, we show the reconstructed images for three different times in Figure 11. It can be seen that something on the order of $12 \mathrm{~h}$ is sufficient, based on an empirical comparison of the spatial variance of an assumed flat background compared to the source distribution.
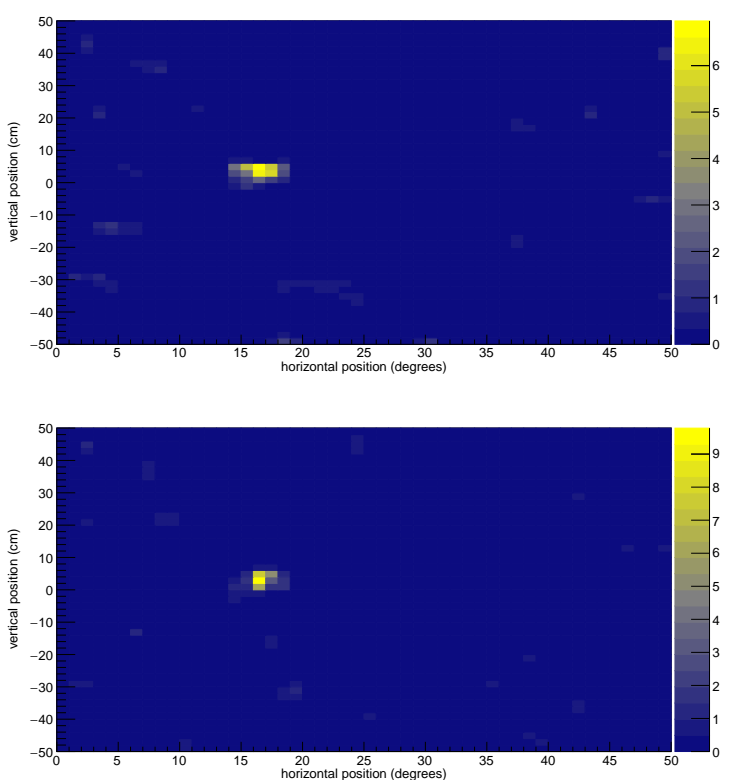

Figure 9: MLEM reconstructions of two ${ }^{252} \mathrm{Cf}$ neutron point sources separated by $2^{\circ}$ at $2.0 \mathrm{~m}$ stand-off. (top) $24 \mathrm{~h}$ dwell time and 250 MLEM iterations. (bottom) Same positions as on the top, but only $2 \mathrm{~h}$ dwell time and 100 MLEM iterations. The $\mathrm{z}$-axis is the rate of neutrons in $\mathrm{Hz}$. 

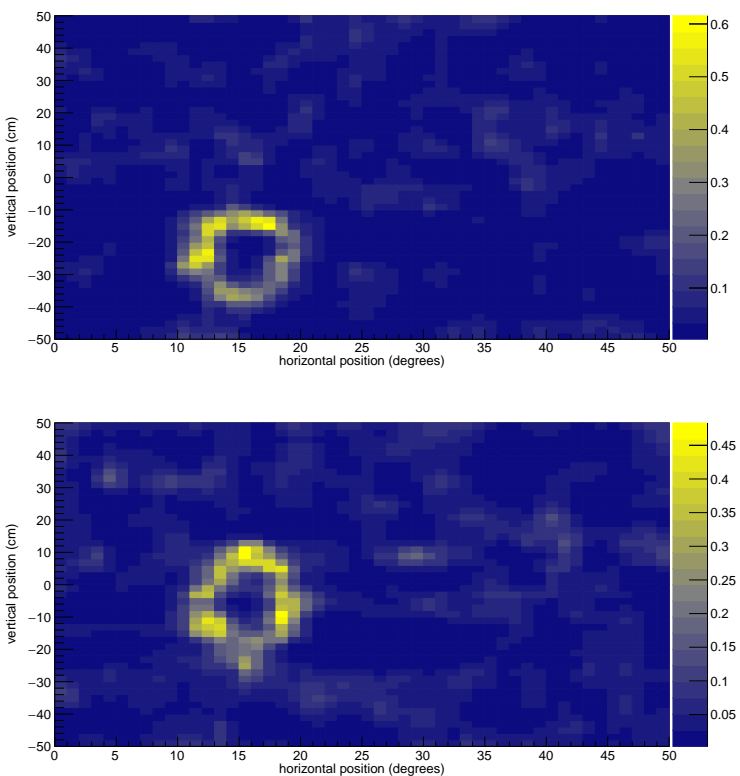

Figure 10: MLEM reconstruction of a ${ }^{252} \mathrm{Cf}$ ring source in two different locations at $2.0 \mathrm{~m}$ stand-off. The color scale is in units of neutron rate (n/s). $94 \mathrm{~h}$ dwell, 100 MLEM iterations (bottom) and $72 \mathrm{~h}$ dwell, 100 MLEM iterations (top). The apparent angular diameter of the source is expected to be $8.7^{\circ}$. The $\mathrm{z}$-axis is the rate of neutrons in $\mathrm{Hz}$.

These images also indicate that, because of the cylin- drical geometry of the mask, there are artifacts that emerge as horizontal stripes in the reconstruction, presumably leading to the image distortions visible in Figures 10 and 11 . We have identified several possible reasons for this. First, the horizontal modulation is achieved through continuous rotation of the mask and therefore lends itself to a smooth reconstruction. Vertically, variations in the mask from row to row can lead to different performance for different horizontal bands across the field of view of the imager. This could be addressed by either introducing a vertical oscillation to the detectors, or changing to a spherical geometry without a single fixed rotation axis (although such a mask would be difficult to engineer). Second, not every row of the mask pattern has the same open fraction, causing different horizontal bands in the field of view to have different efficiencies. This could be addressed with better optimization of the mask, or it could be corrected by dividing out the vertical dependence of the efficiency. Third, different rows of the mask have their own independent modulation performance. Since the mask was not well optimized, some rows may perform better than others. Better optimization of the mask through a heuristic process that converges toward the best performance would
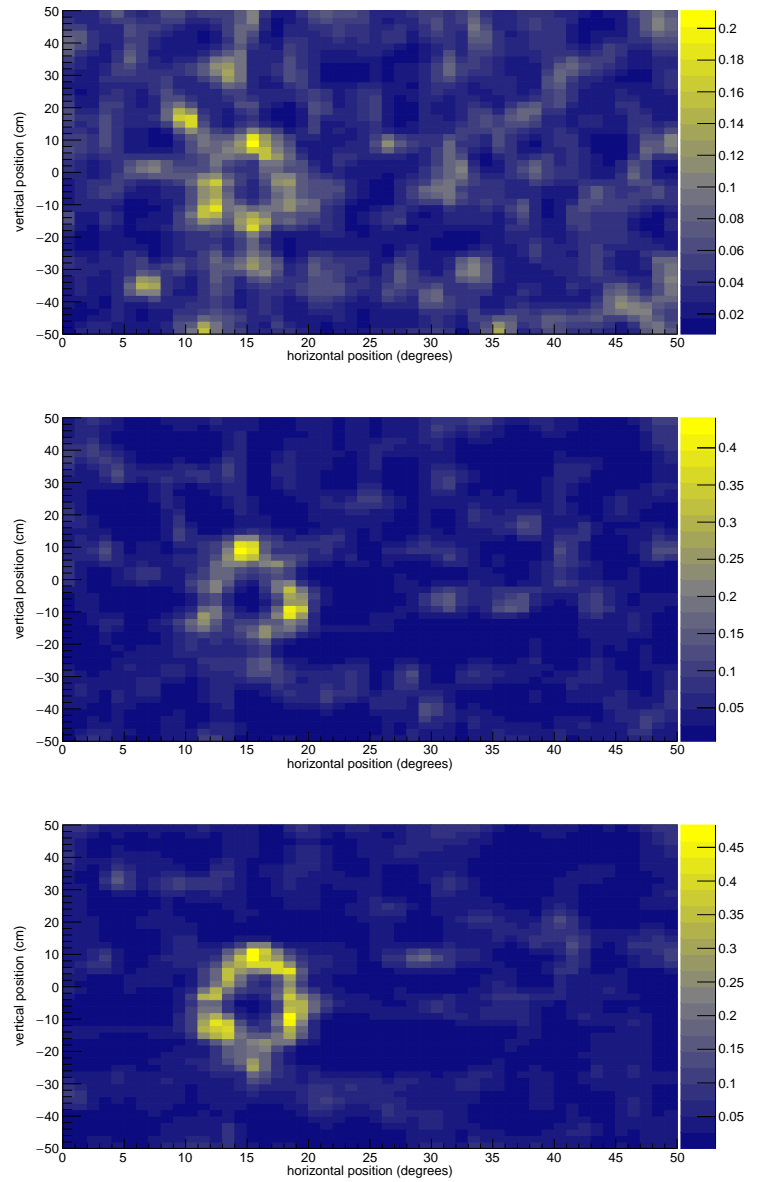

Figure 11: MLEM reconstruction of the same ring source with different dwell times. (top) $3 \mathrm{~h}, 20$ MLEM iterations, (middle) $12 \mathrm{~h}, 50$ MLEM iterations, (bottom) $72 \mathrm{~h}, 100$ MLEM iterations. The $\mathrm{z}$-axis is the rate of neutrons in $\mathrm{Hz}$. 
reduce this effect [6]. Finally, for regions of the field of 391 view with a line of sight to the liquid scintillator detectors that split two mask rows, the average modulation ${ }_{393}^{392}$ will be quite poor. For these regions, one would expect ${ }_{394}$ to find on average one mask element and one aperture 395 at any given rotation angle, leading to the poor mod- ${ }^{396}$ ulation. We understood that this would be a problem ${ }^{397}$ in designing this system, and therefore added a second ${ }_{399}$ liquid scintillator detector with vertical separation of an 400 integer number plus one half the height of a mask ele- ${ }^{401}$ ment. This was done so that there would not be regions ${ }^{402}$ of the field of view that have this modulation problem ${ }^{404}$ for both detectors simultaneously. It is conceivable that 405 a detector with vertical position resolution, such as a ${ }^{406}$ bar type geometry with a PMT on each end, could bring continuity to the reconstruction vertically.

\section{Discussion and Conclusions}

Through a series of laboratory measurements on a variety of test objects, we have demonstrated the feasibil- ${ }^{417}$ ity of two-dimensional fast neutron imaging using the ${ }^{418}$ time modulation of rates on a single pixel detector. Two point sources with $2^{\circ}$ separation were distinguishable from a single point source in $12 \mathrm{~h}$. Extended source distributions were also successfully imaged, although there were some performance issues which will be addressed in future analysis and tests.

The simplicity of analyzing a single time dependent neutron rate from a single neutron detector may make this an attractive option for applications that demand high resolution mapping of a radiation field without terribly strict time restrictions, such as arms control and treaty verification. To our knowledge, this is the first investigation of 2D-TEI for nuclear nonproliferation applications.

\section{Acknowledgments}

Sandia National Laboratories is a multi-program laboratory managed and operated by Sandia Corporation, a wholly owned subsidiary of Lockheed Martin Corporation, for the U.S. Department of Energy's National Nuclear Security Administration under contract DE-AC0494AL85000. Document release number SAND2015$6244 \mathrm{~J}$.

We thank the US DOE National Nuclear Security Administration, Defense Nuclear Non-Proliferation Office of Research and Development for funding this work.

\section{References}

[1] J. Brennan, E. Brubaker, R. Cooper, M. Gerling, C. Greenberg, P. Marleau, N. Mascarenhas and S. Mrowka. "Measurement of the Fast Neutron Energy Spectrum of an ${ }^{241}$ Am-Be Source Using a Neutron Scatter Camera" IEEE Transactions on Nuclear Science 58 (2011) 2426-2430

[2] N. Mascarenhas, J. Brennan, K. Krenz, P. Marleau, and S. Mrowka. "Results with the Neutron Scatter Camera" IEEE Transactions on Nuclear Science 56 (2009) 1269-1273

[3] P. Hausladen, M. Blackston, E. Brubaker, D. Chichester, P. Marleau, and J. Newby. "Fast Neutron Coded-Aperture Imaging of Special Nuclear Material Configurations INMM Conference Proceedings (2012)

[4] S.R. Gottesman and E.E. Fenimore "New family of binary arrays for coded aperture imaging" Applied Optics 28 (1989)

[5] E.E. Fenimore and T.M. Cannon. "Coded Aperture Imaging with Uniformly Redundant Arrays" Applied Optics 17 (1978)

[6] P. Marleau, M. Blackston, J. Brennan, E. Brubaker, M. Gerling, P. Hausladen, K. McMillan, S. Mrowka, and J. Newby, "High Resolution Imaging of Fission Energy Neutrons." INMM Conference Proceedings (2012)

[7] L. Shepp and Y. Vardi. "Maximum Likelihood Reconstruction for Emission Tomography." IEEE Transactions on Medical Imaging 1 (1982) 113-122.

[8] R. Brun and F. Rademakers. Nucl. Inst. and Meth. in Phys. Res. A 389 (1997) 81-86

[9] E. Caroli, J.B. Stephen, G. Di Cocco, L. Natalucci, and A. Spizzichino. "Coded Aperture Imaging in X- and Gamma-Ray Astronomy." Space Science Reviews 45 (1987) 349-403. 
$2-d$ coded mask

Single 1"D $x 1^{\prime \prime}$ LS pixel
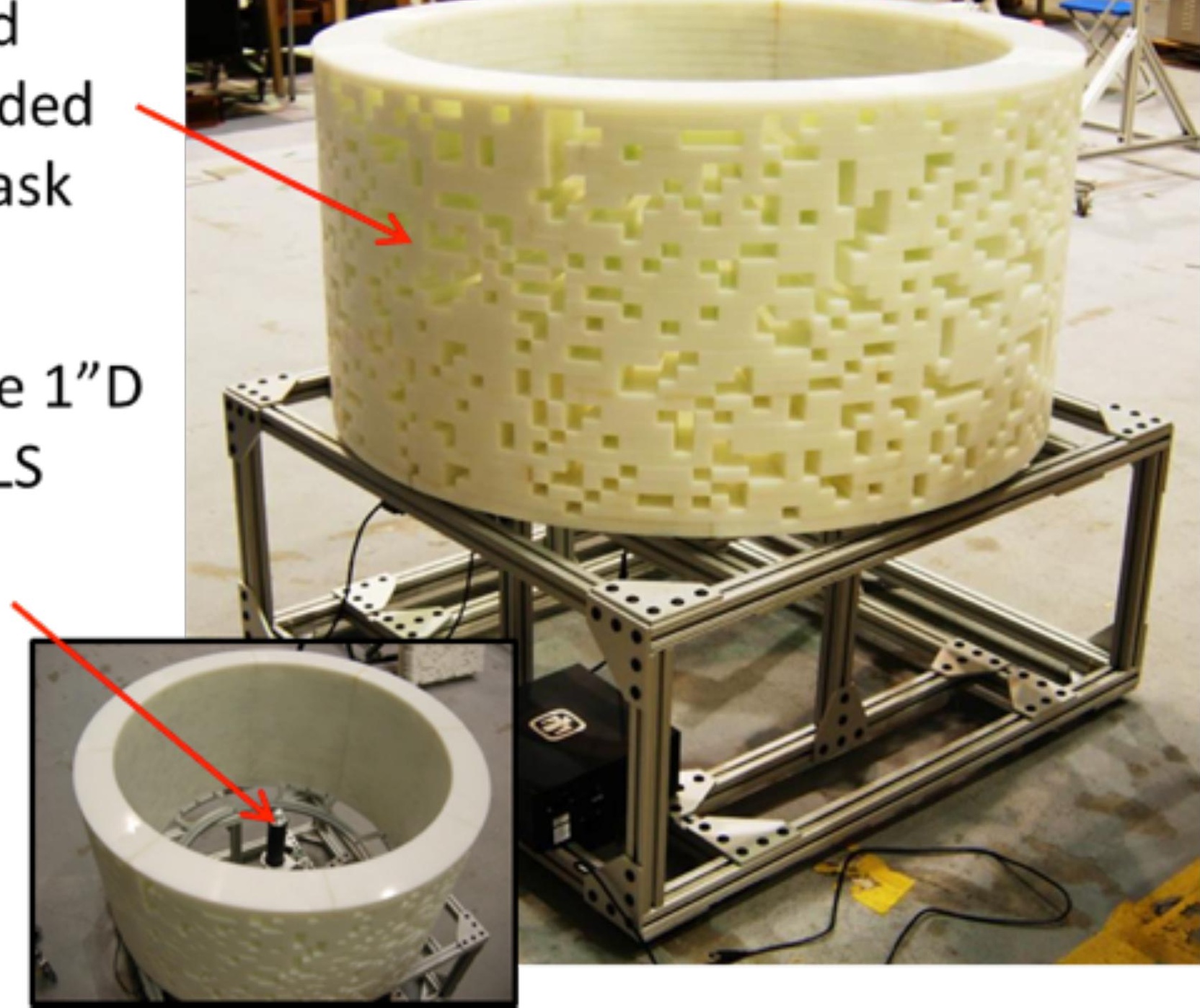

Figure
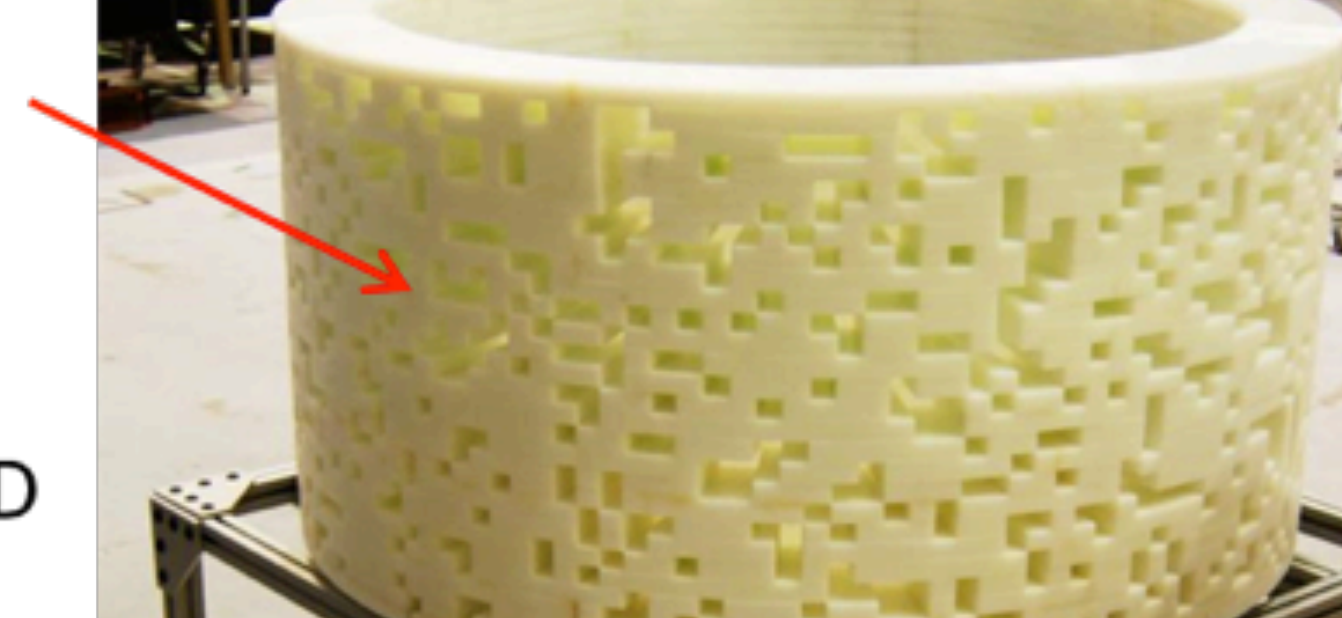
figure 2

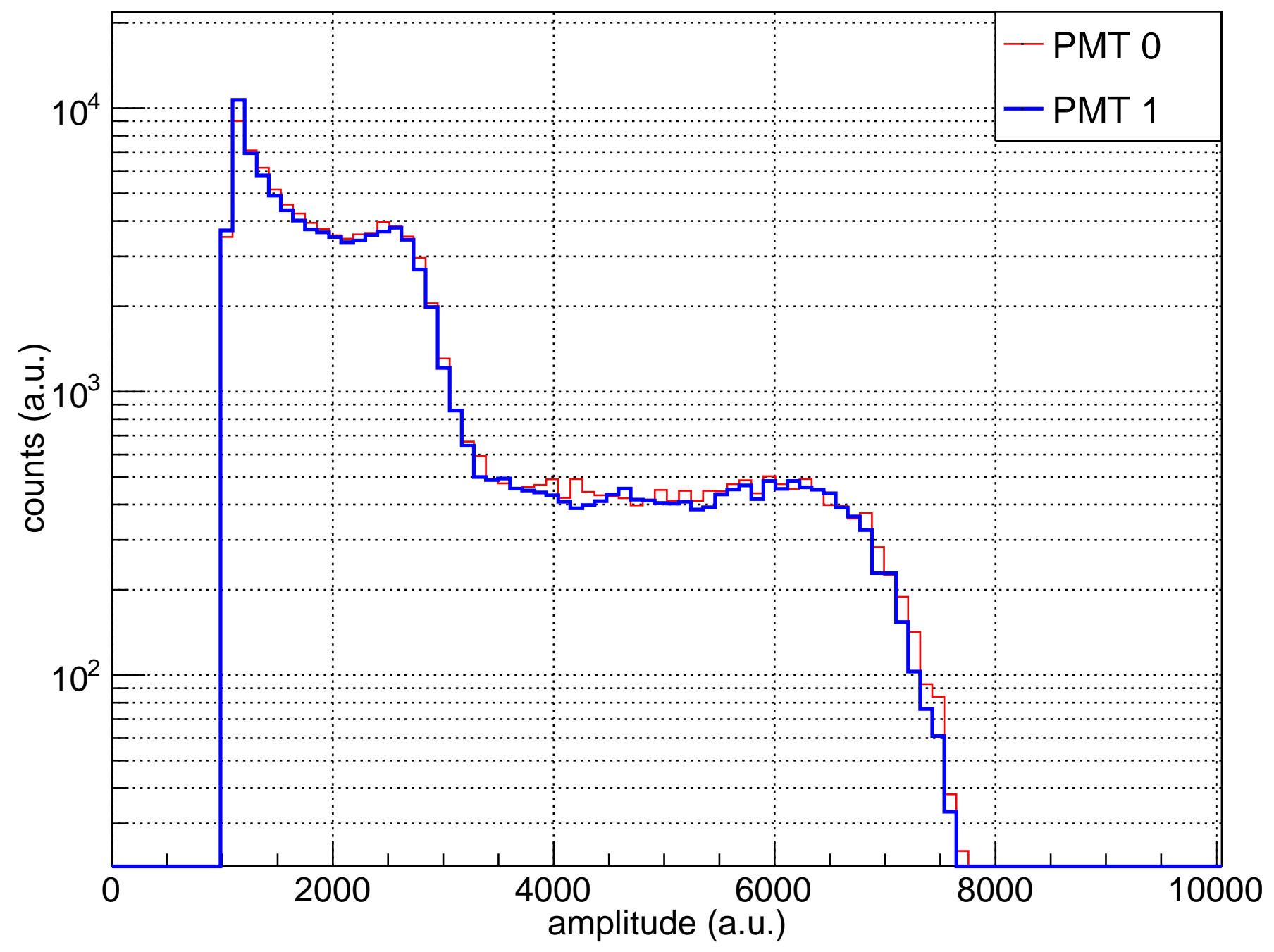


figure 3

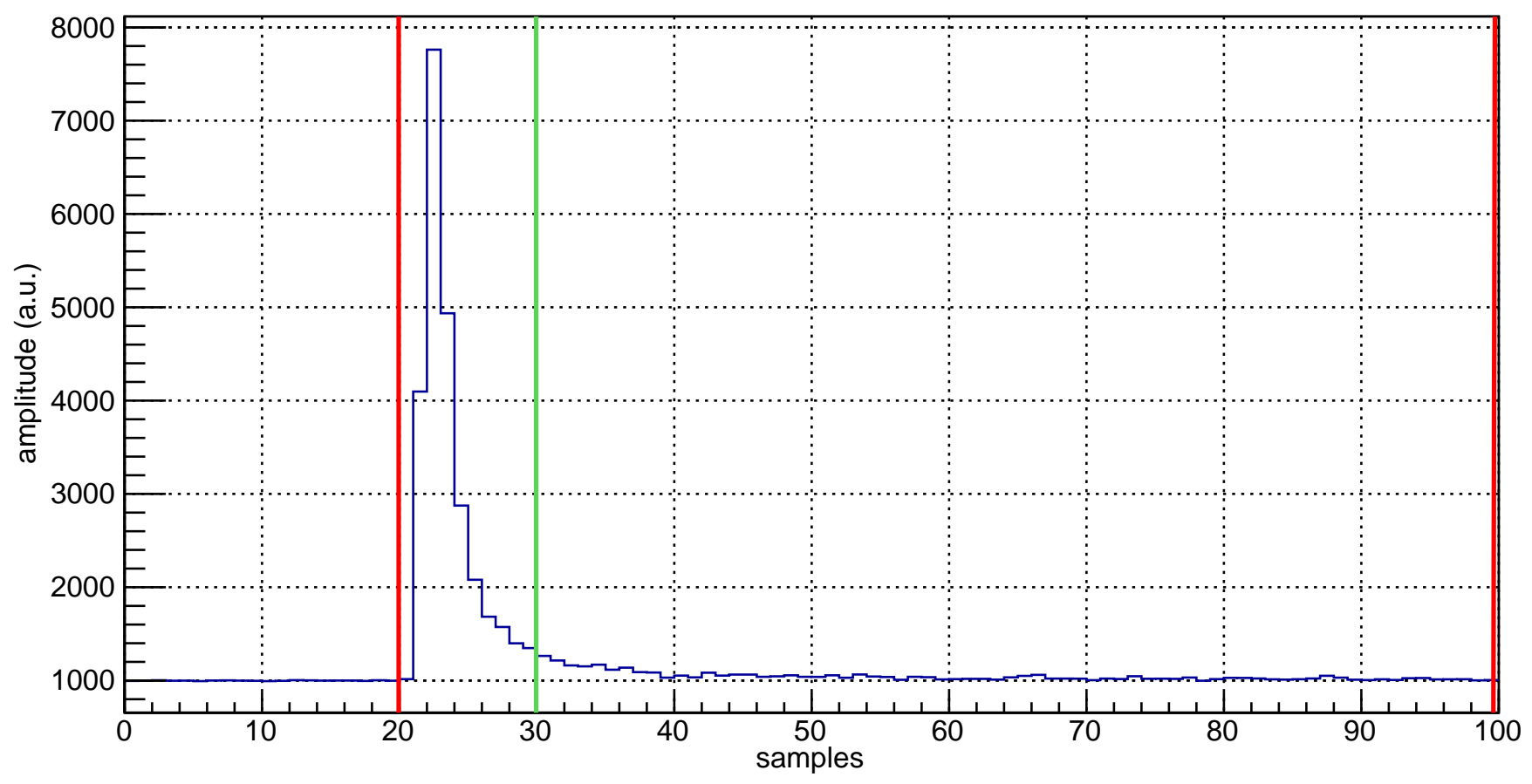


figure $4 a$

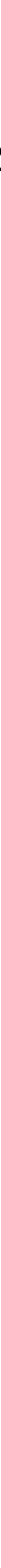


figure $4 b$

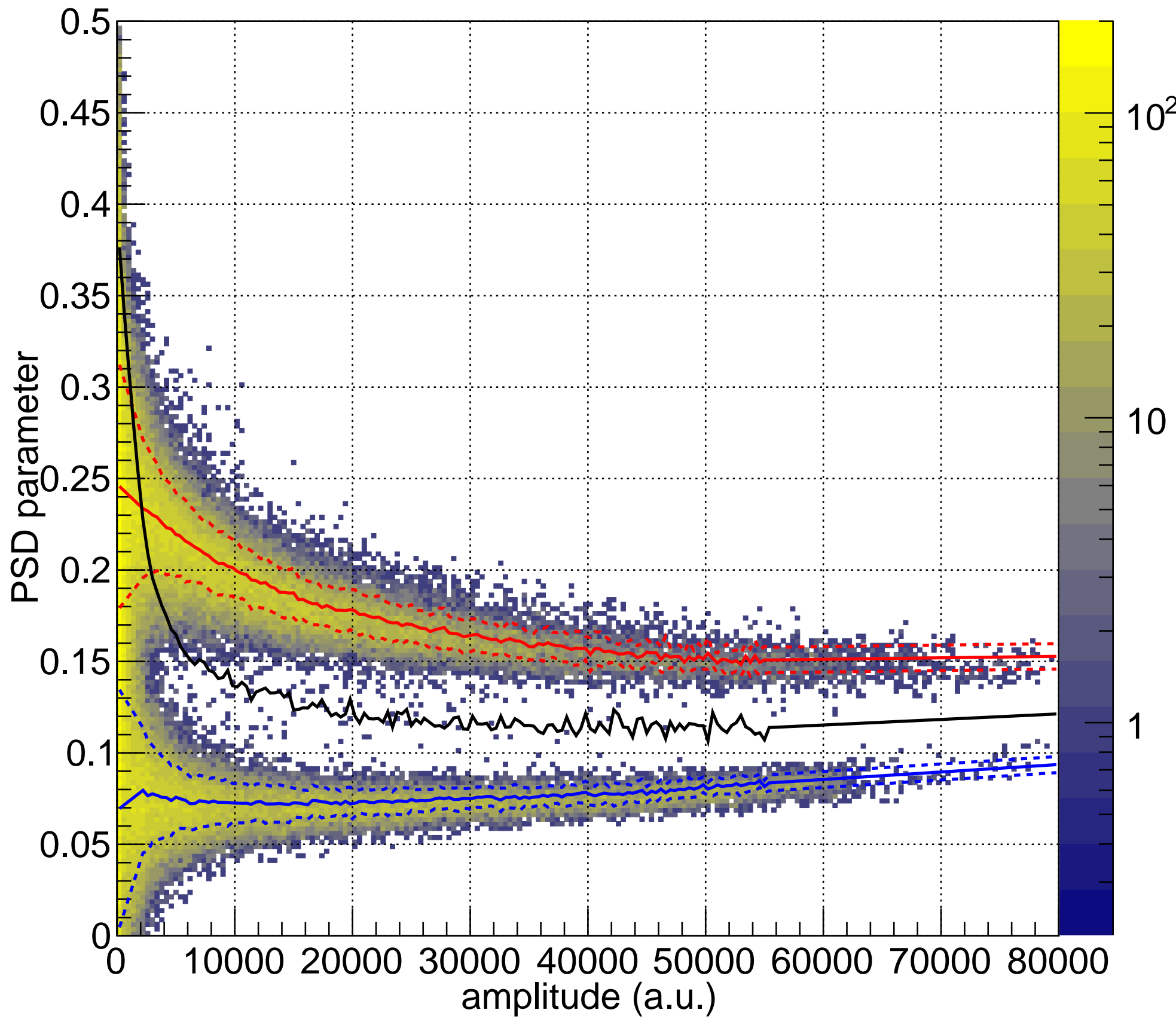




\section{figure 5}

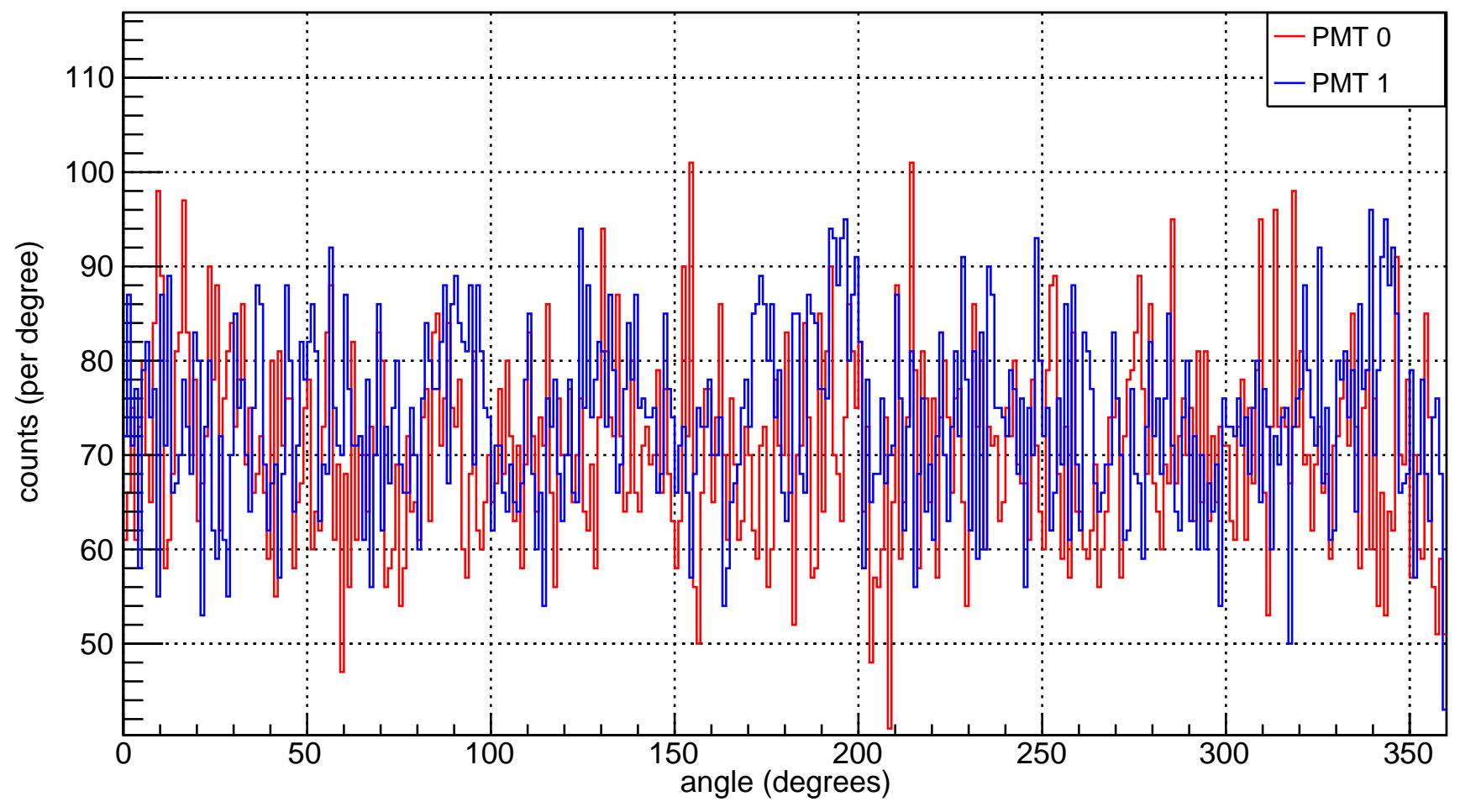


figure 6

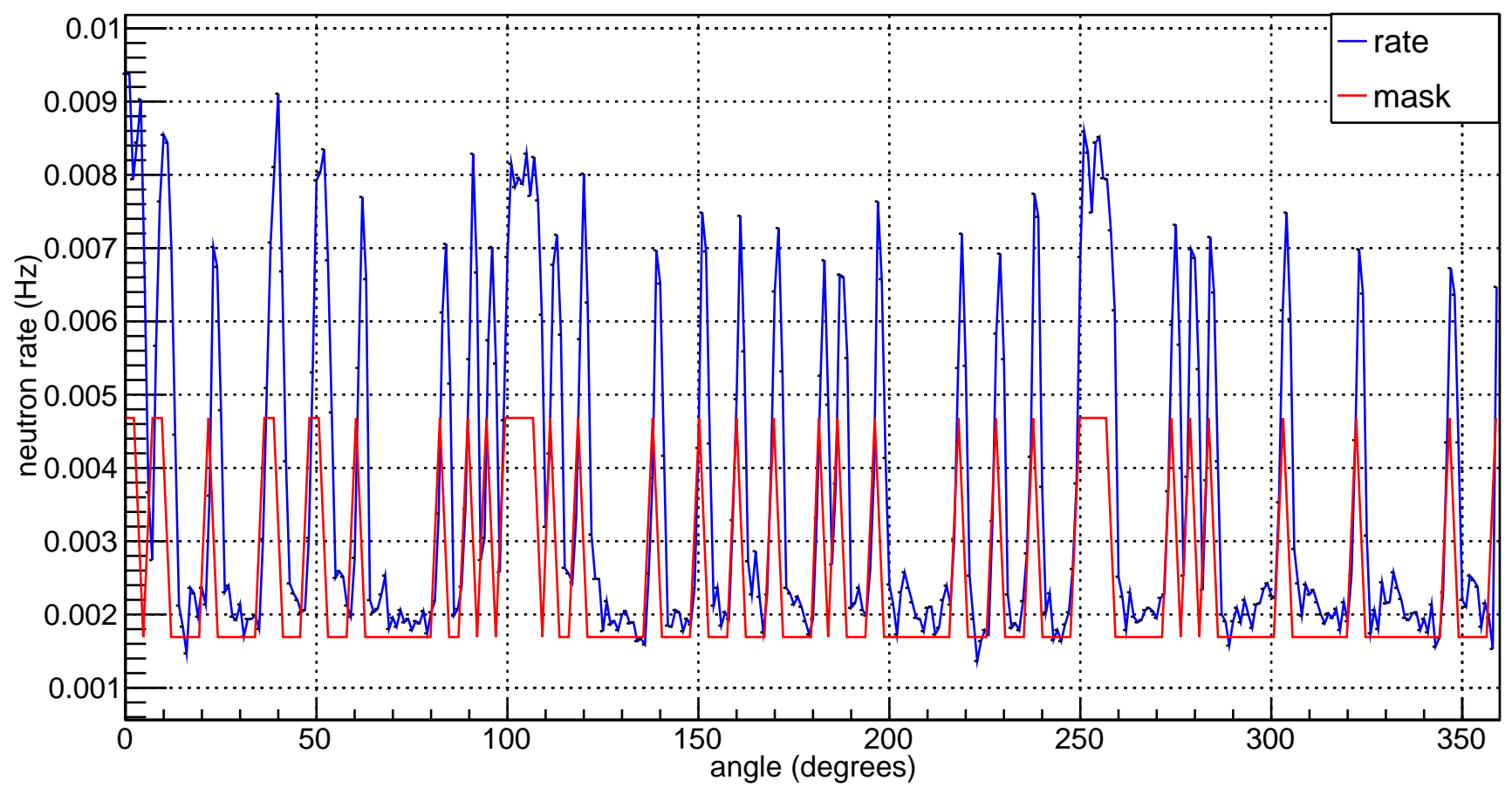


figure 7

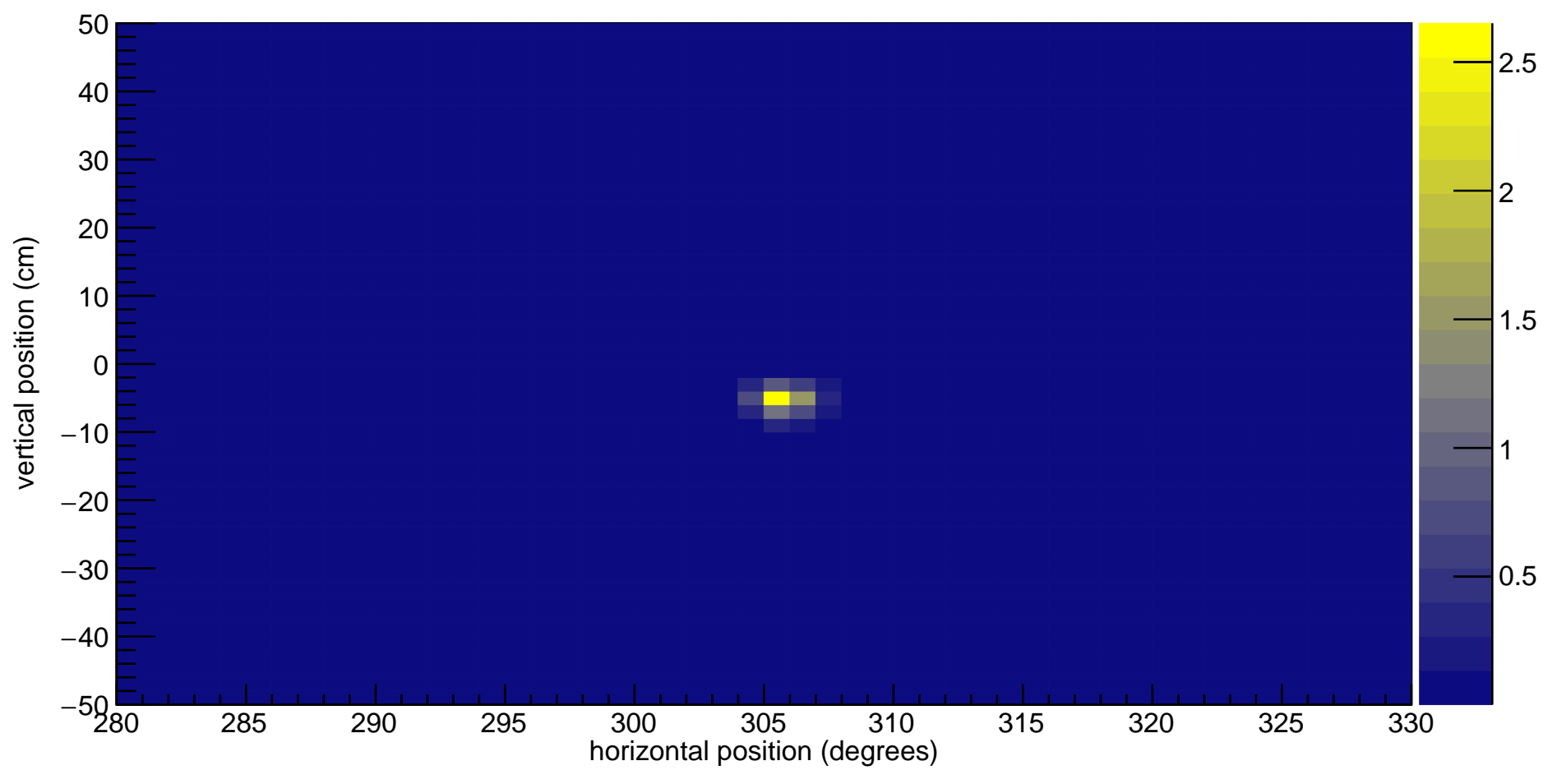


figure $8 a$

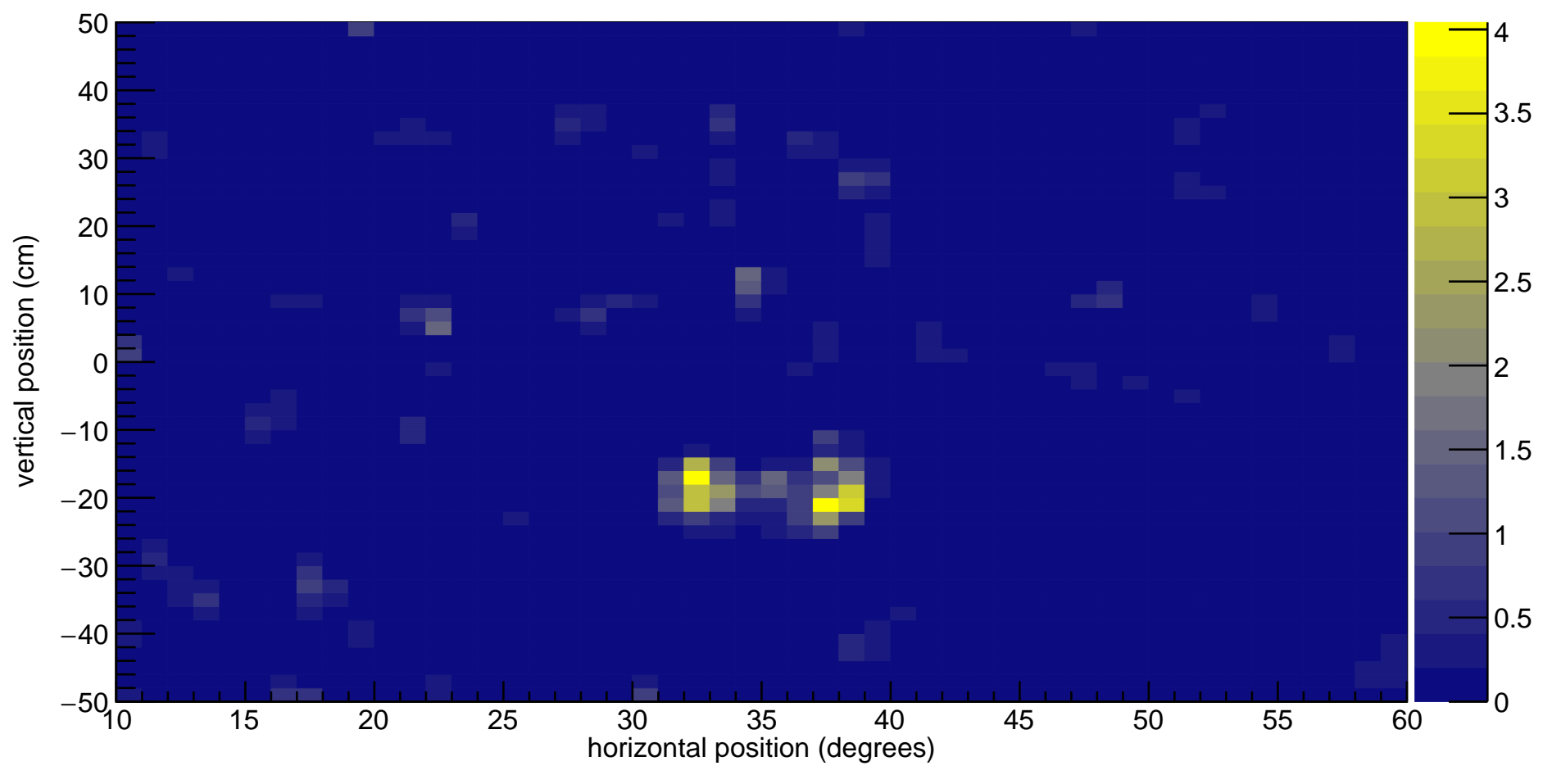




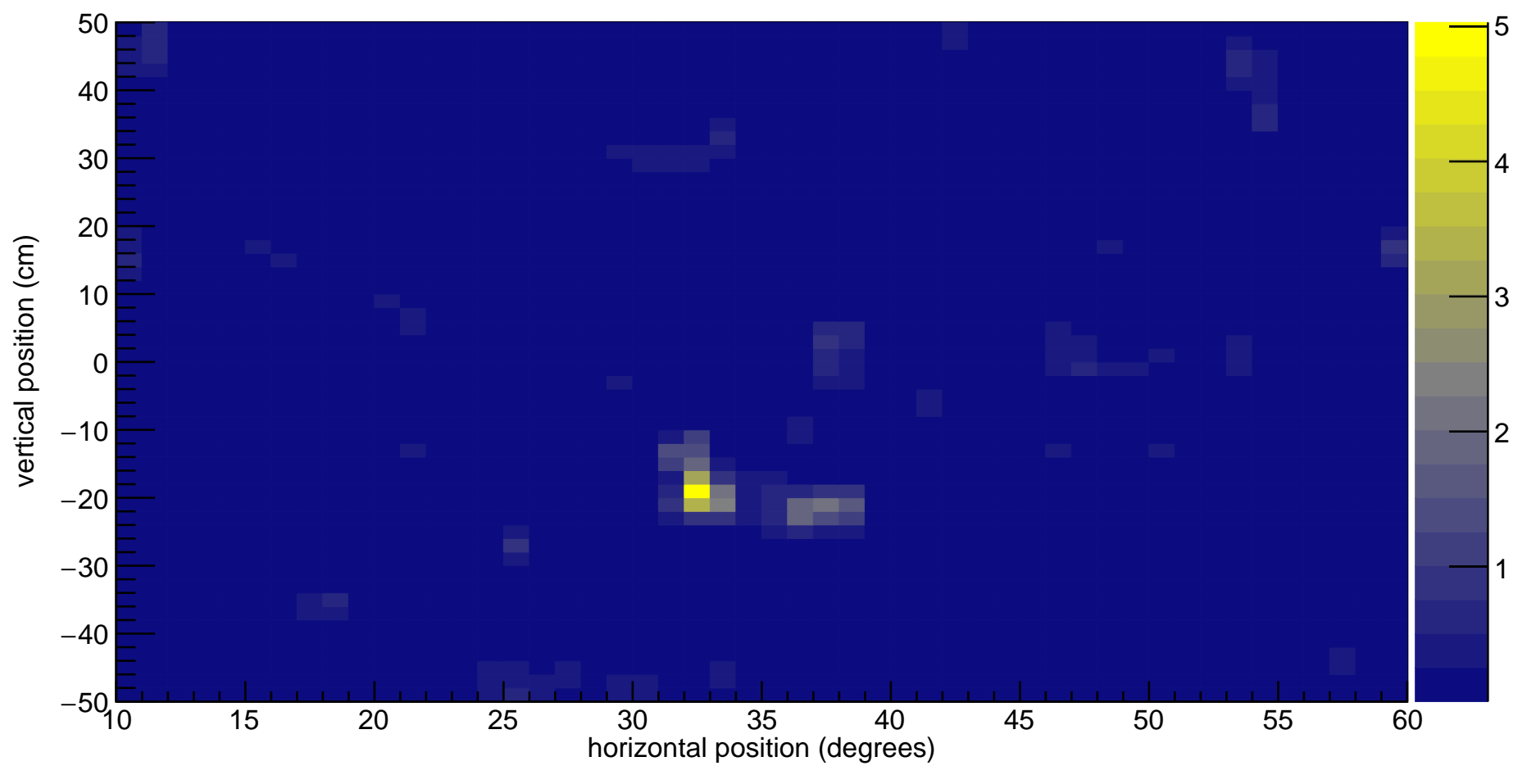


figure $8 c$

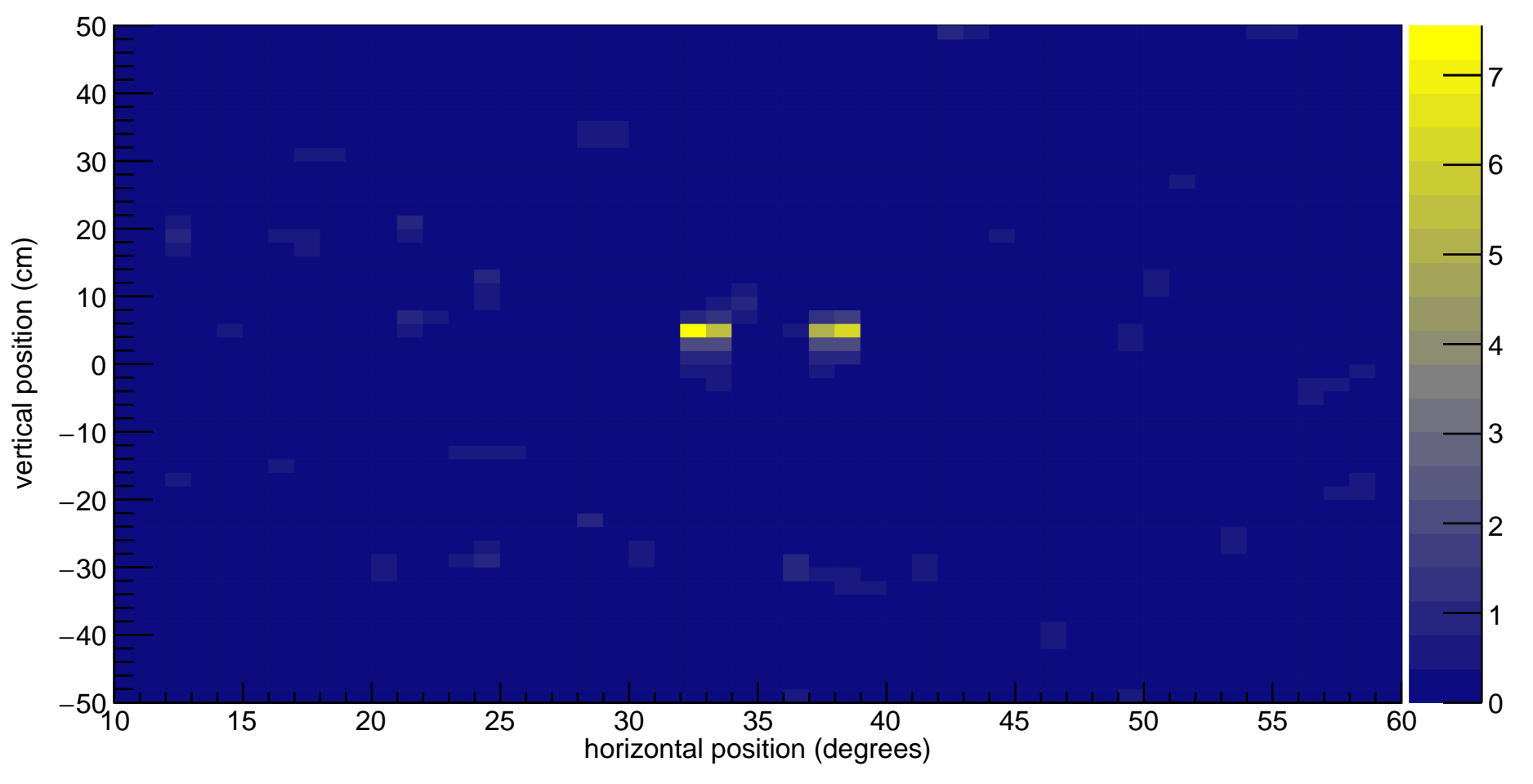


figure $8 d$

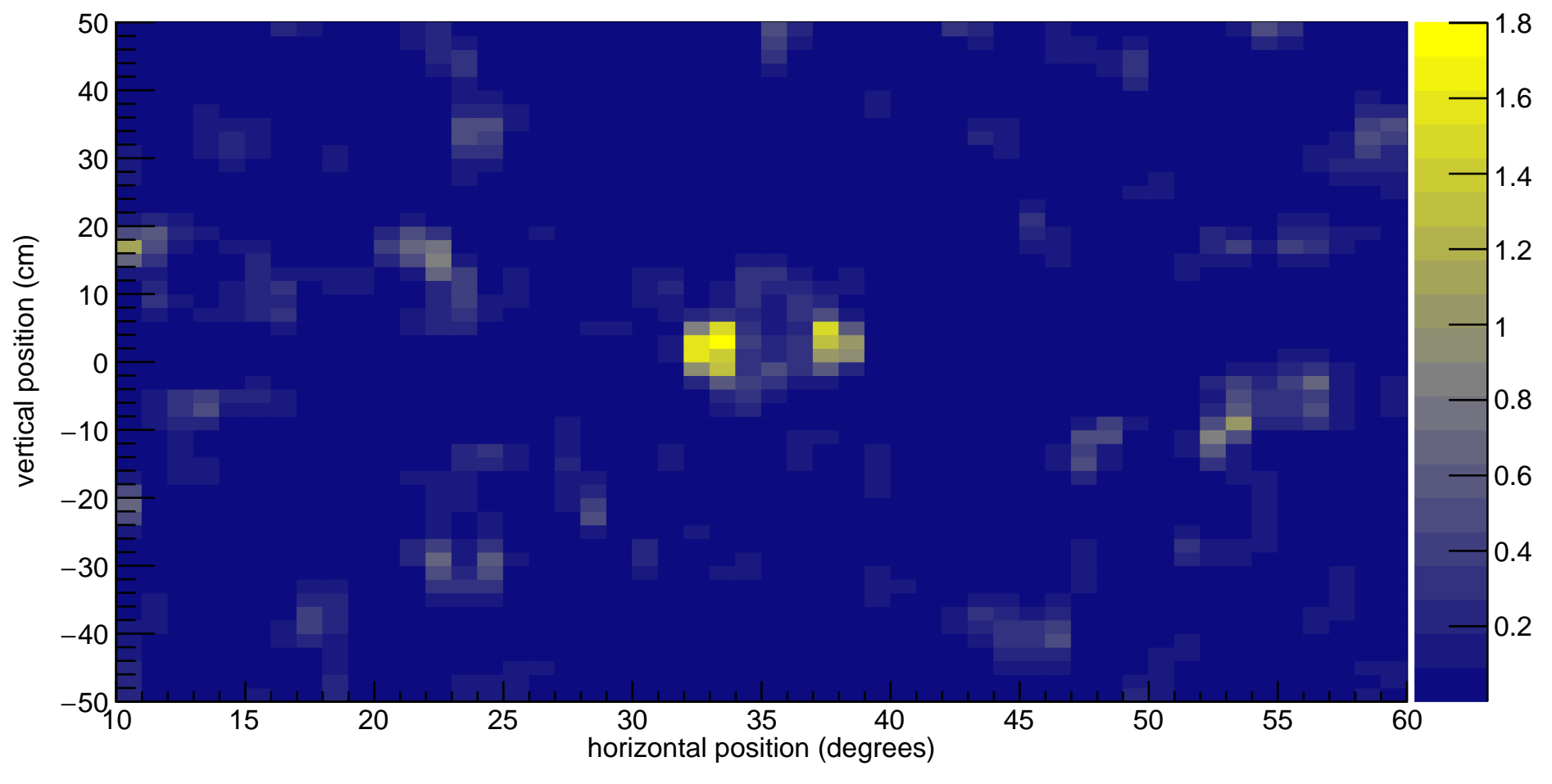


figure $9 a$

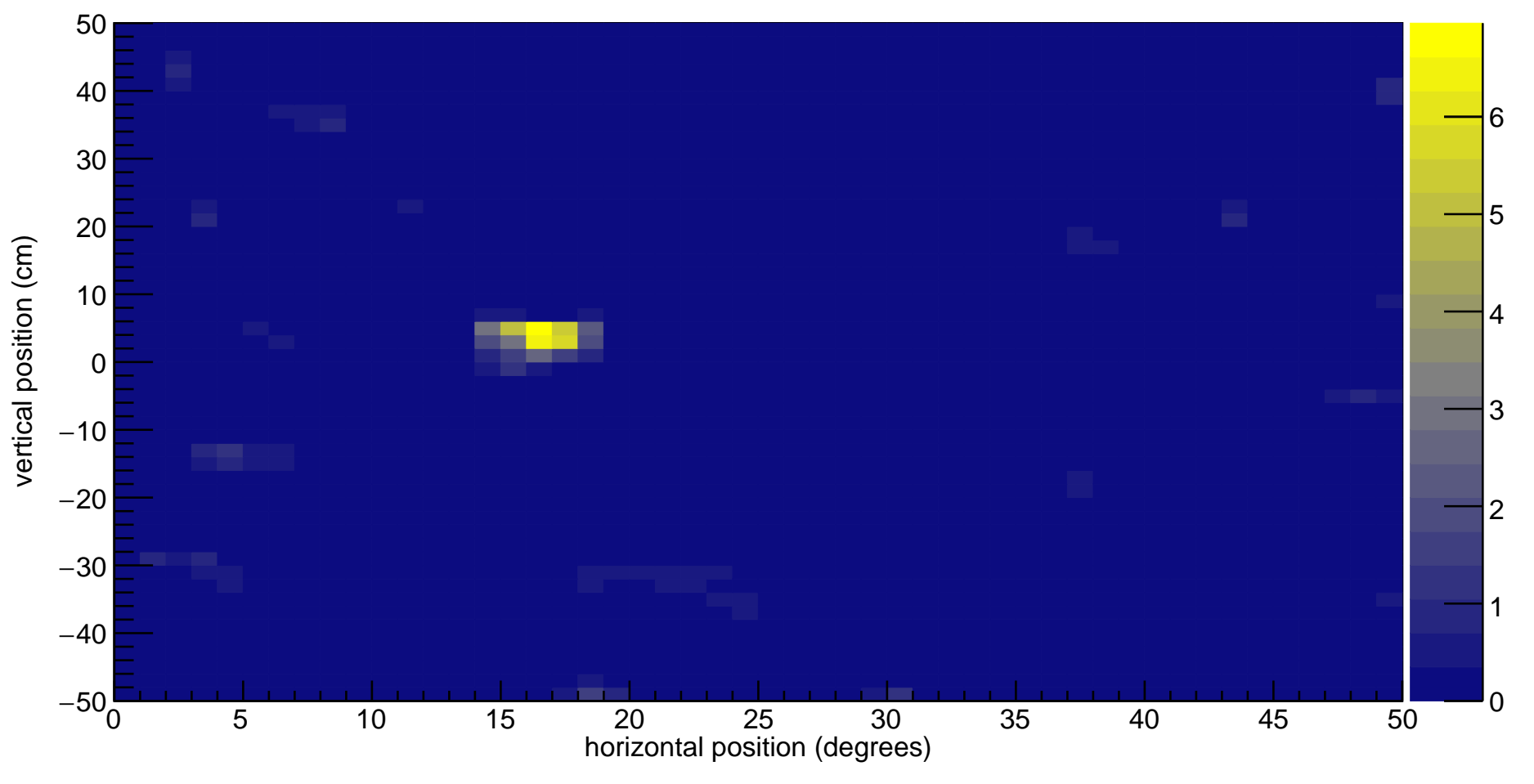




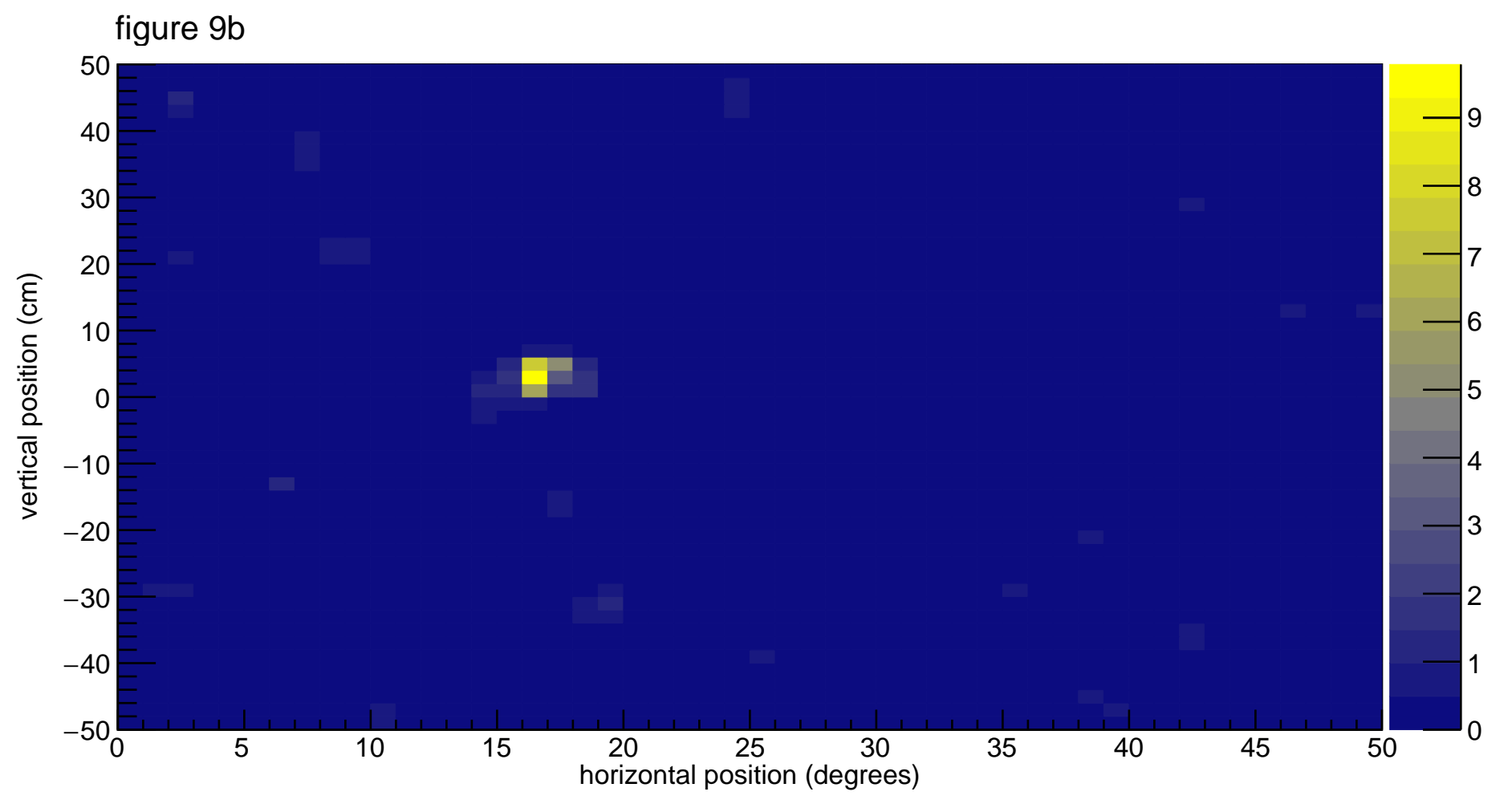


figure $10 a$

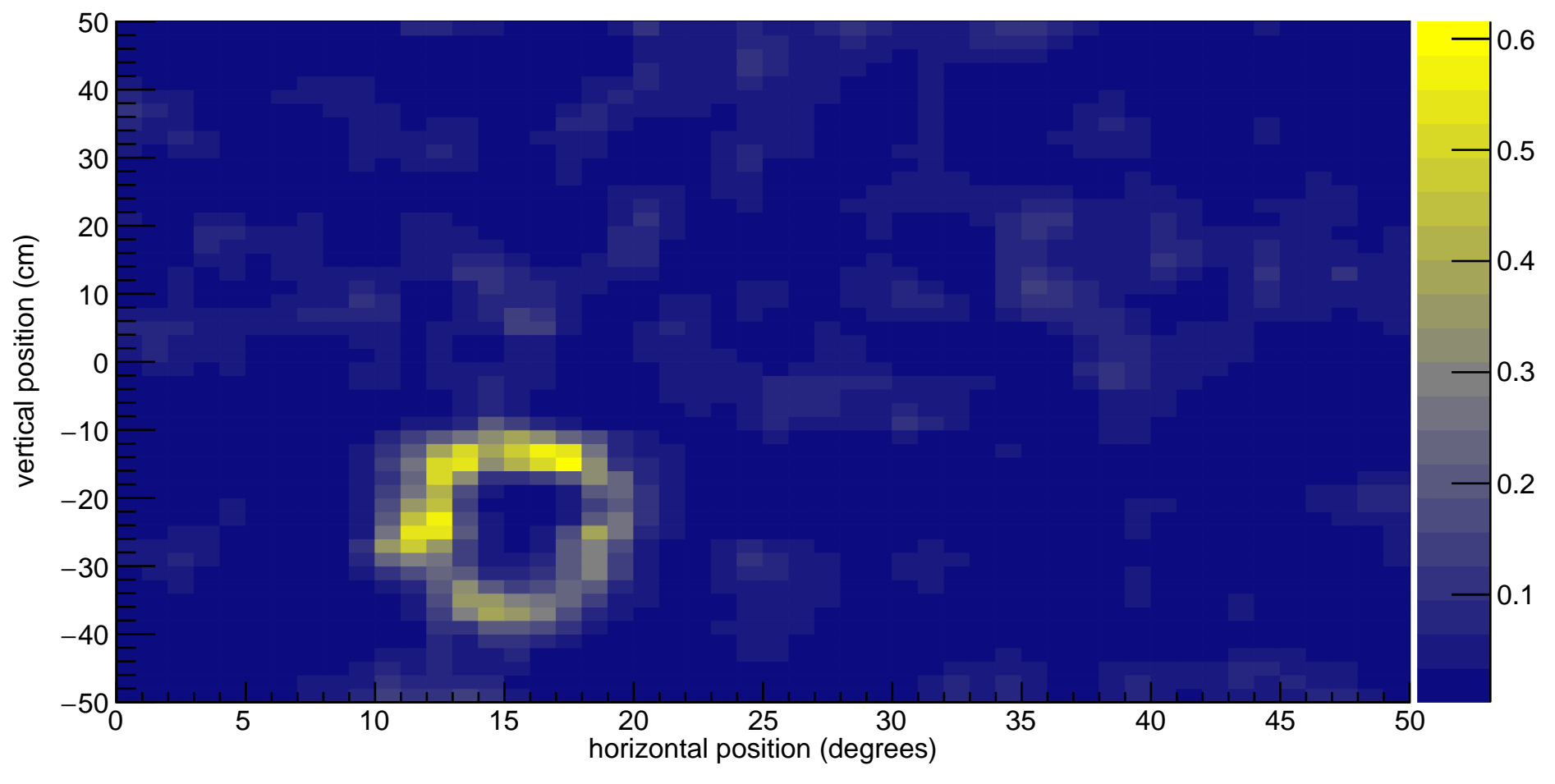




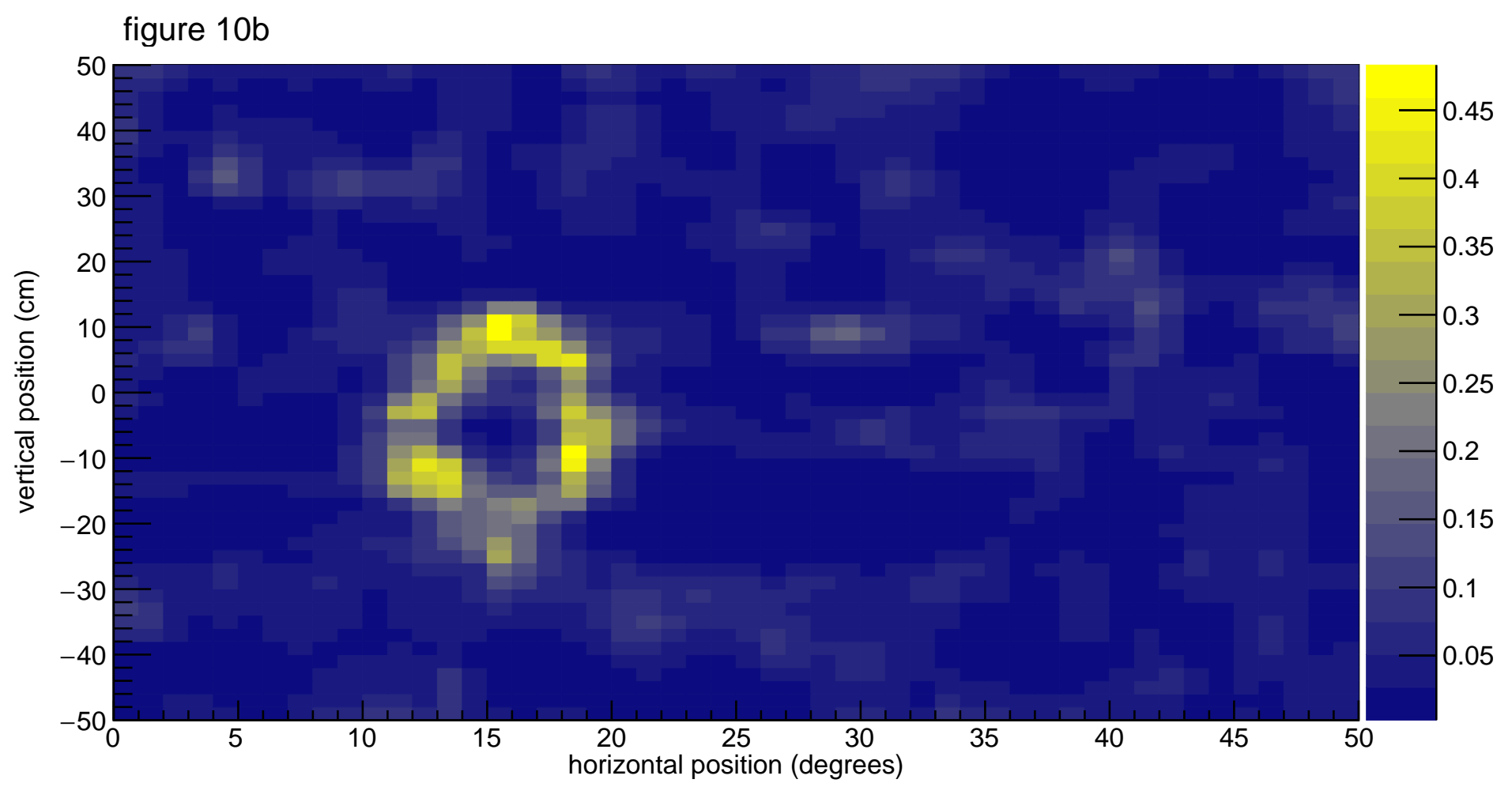


figure $11 a$

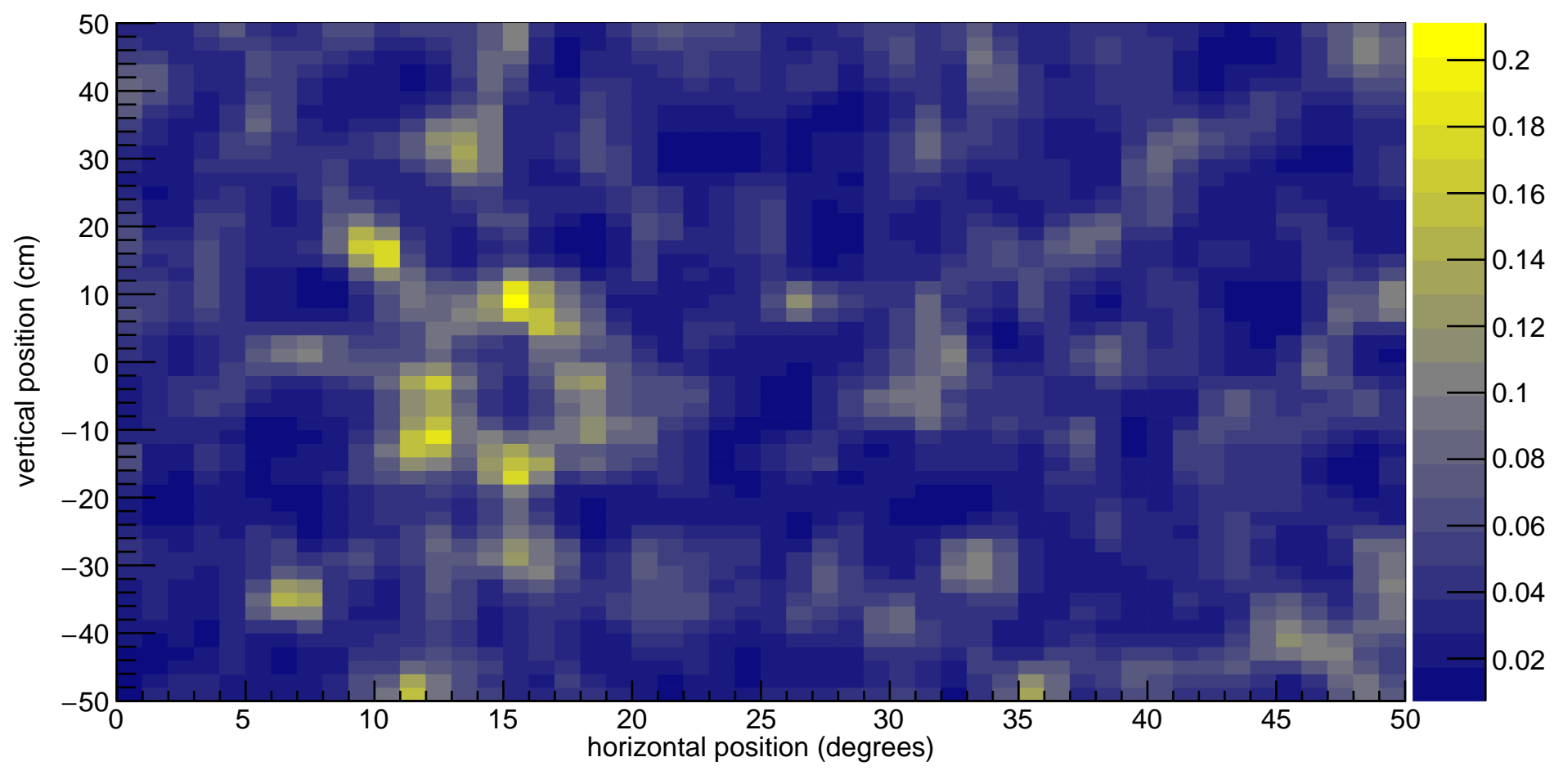




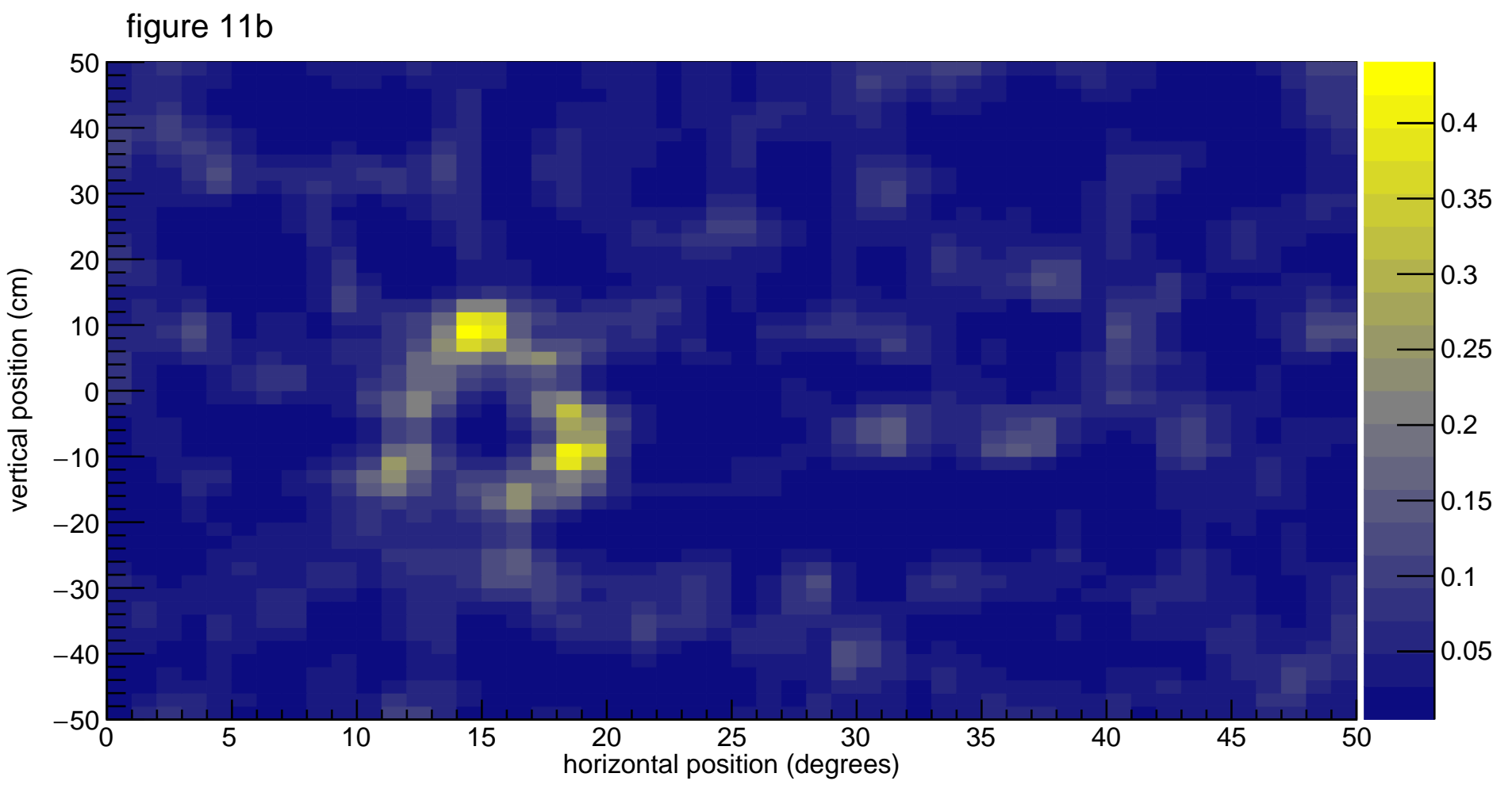


figure 11c

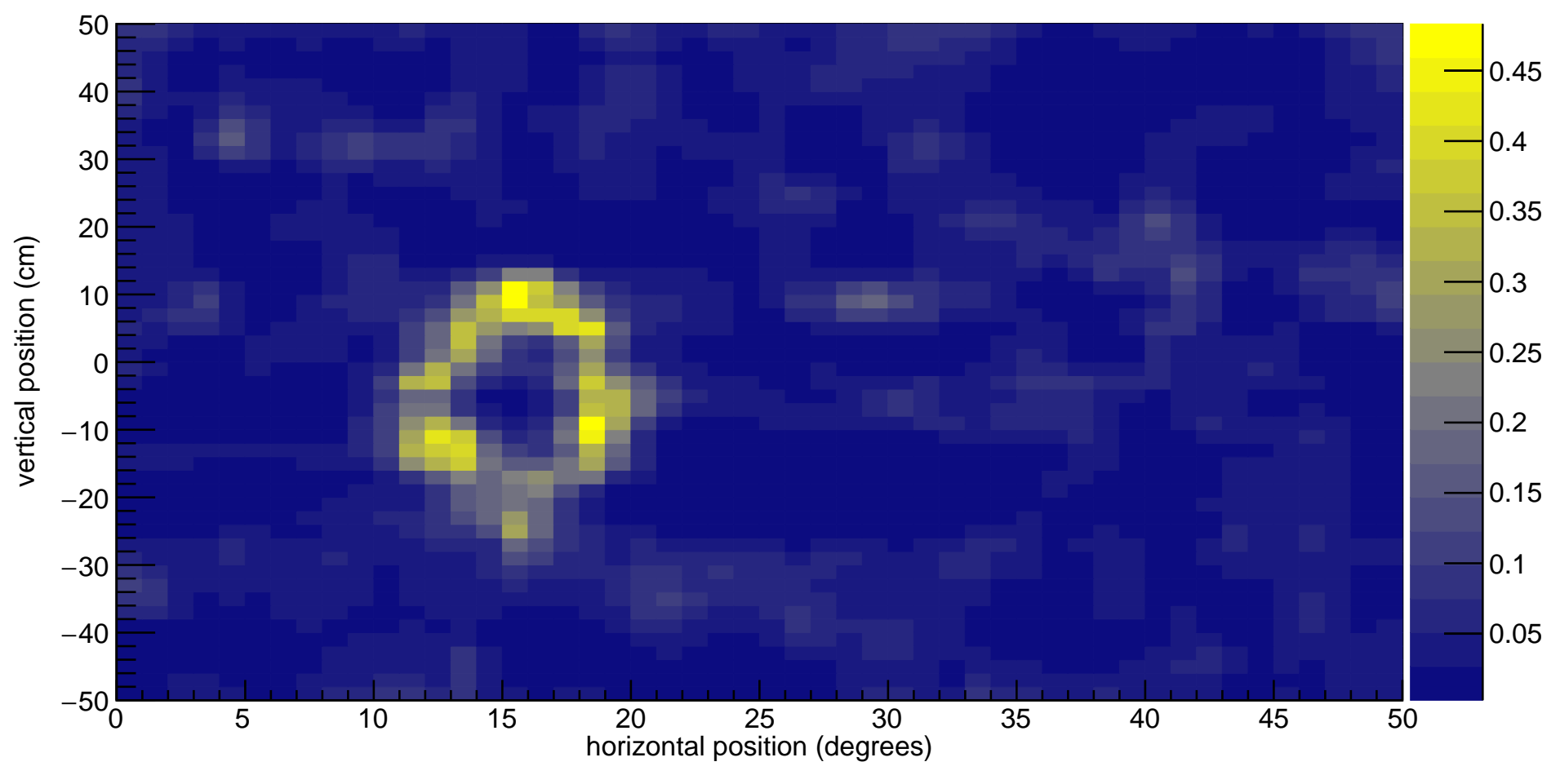

